\title{
Exoplanets in binary star systems: on the switch from prograde to retrograde orbits
}

\author{
J. P. S. Carvalho ${ }^{1}$ • D. C. Mourão ${ }^{4}$ - R. Vilhena de Moraes ${ }^{2}$. \\ A. F. B. A. Prado ${ }^{3}$. O. C. Winter ${ }^{4}$
}

Received: 29 April 2014 / Revised: 6 July 2015 / Accepted: 30 August 2015 /

Published online: 8 October 2015

C Springer Science+Business Media Dordrecht 2015

\begin{abstract}
The eccentric Kozai-Lidov mechanism, based on the secular theory, has been proposed as a mechanism that plays an important role in producing orbits that switch from prograde to retrograde. In the present work we study the secular dynamics of a triple system composed of a Sun-like central star and a Jupiter-like planet, which are under the gravitational influence of another perturbing star (brown dwarf). The perturbation potential is developed in closed form up to the fifth order in a small parameter $\left(\alpha=a_{1} / a_{2}\right)$, where $a_{1}$ is the semimajor axis of the extrasolar planet and $a_{2}$ is the semimajor axis of the perturbing star. To eliminate the short-period terms of the perturbation potential, the double-average method is applied. In this work we do not eliminate the nodes, a standard method in the literature, before deriving the equations of motion. The main goal is to study the effects of the higher-order terms of the expansion of the perturbing force due to the third body in the orbital evolution of the planet. In particular, we investigate the inclination and the shape (eccentricity) of these orbits. We show the importance of the higher-order terms in changing the inversion times of the
\end{abstract}

$\bowtie \quad$ J.P. S. Carvalho

jeanfeg@gmail.com

D. C. Mourão

decmou@yahoo.com.br

R. Vilhena de Moraes

rodolpho.vilhena@gmail.com

A. F. B. Prado

antonio.prado@inpe.br

O. C. Winter

ocwinter@gmail.com

1 Centro de Ciência e Tecnologia em Energia e Sustentabilidade, Universidade Federal do Recôcavo da Bahia (UFRB), Feira de Santana, BA CEP: 44085-132, Brazil

2 Instituto de Ciência e Tecnologia, Universidade Federal de São Paulo (UNIFESP), São José dos Campos, SP CEP: 12231-280, Brazil

3 Division of Space Mechanics and Control, INPE, São José dos Campos, SP CEP: 12227-010, Brazil

4 Univ Estadual Paulista (UNESP), Guaratinguetá, SP CEP: 12516-410, Brazil 
flip, i.e., the times where the inclination of the inner planet flips from prograde to retrograde trajectories. We also show the dependence of the first flip with respect to the semimajor axis and eccentricity of the orbit of the planet. The general conclusion is that the analytical model increases its accuracy with the inclusion of higher-order terms. We also performed full numerical integrations using the Bulirsch-Stoer method available in the Mercury package for comparison with the analytical model. The results obtained with the equations developed in this work are in accordance with direct numerical simulations.

Keywords Three-body problem - Orbital perturbation · Exoplanets · Flip of inclination · Lidov-Kozai mechanism

\section{Introduction}

So-called hot Jupiters are extrasolar planets with a mass similar to that of Jupiter and have orbits that are very close to the central star. The sky-projected angle between the orbits of several hot Jupiters and the spins of their host stars were measured, and the results indicated that misalignment and even retrograde orbits are common (Triaud et al. 2010; Albrecht et al. 2012). More than $20 \%$ of these planets are found in retrograde orbits with respect to the spin's angular momentum of the host star. The eccentric Kozai-Lidov mechanism (Kozai 1962; Lidov 1962), based on secular theory, has been proposed as a mechanism that plays an important role in producing such orbits (Naoz et al. 2011, 2012, 2013). In the present work we study the secular dynamics of a triple system composed of a Sunlike central star and a Jupiter-like planet, which are under the gravitational influence of another perturbing star (brown dwarf). Naoz et al. (2011) presented a study in which they take into account the octupole term in the perturbation potential. They show that the inclination of the inner planet varies from prograde $\left(i<90^{\circ}\right)$ to retrograde $\left(i>90^{\circ}\right)$ for a specific problem, where $i$ is the mutual inclination between the two orbits. The authors show that, considering only the quadrupole term in the potential, the inclination varies according to the Kozai-Lidov mechanism (Kozai 1962; Lidov 1962) (Fig. 1 in Naoz et al. 2011), i.e., the inclination oscillates with large amplitudes when the initial inclination has a value larger than the critical inclination $\left(39.23^{\circ}\right)$ and small amplitudes when the initial inclination has a value smaller than this critical inclination. However, the orbit always remains prograde. When the researchers consider the octupole term in the potential, the inclination grows significantly and can flip from prograde to retrograde trajectories. Therefore, the authors show that the octupole term should be considered in the perturbation potential. This mechanism is defined by Lithwick and Naoz (2011) as an eccentric Kozai mechanism (EKM). In Katz et al. (2011) this problem is calculated analytically for high inclinations. The authors also show that the inclination increases strongly and can flip from prograde to retrograde trajectories when the octupole term in the perturbation potential is taken into account. In Naoz et al. (2013) the authors show that it is possible for the inclination to increase strongly and to flip from prograde to retrograde trajectories when only the $R_{2}$ (quadrupole) term is taken into account. But to obtain these results, the authors did not eliminate nodes (Jefferys and Moser 1966; Kozai 1962) directly in the Hamiltonian, which is generally done in the literature. They eliminate the longitude of the ascending node after deriving the equations of motion. For the third order, the authors mention that it is possible to do that even before deriving the equations of motion, namely, at the level of the Hamiltonian. Then, for the $R_{3}$ term, they eliminate the nodes using the standard method. 
Note that this problem (elimination of nodes) does not appear in the vectorial formalism (e.g., Tremaine et al. 2009; Petrovich 2015a, b), only in the Hamiltonian formalism. Li et al. (2014a) investigated the chaotic and quasiperiodic orbital evolution by studying the surfaces of sections and the Lyapunov exponents (in the test particle limit). The authors found that the resonances introduced by the octupole level of approximation causes the orbits to flip from prograde to retrograde and back and cause significant eccentricity excitations. Chaotic behaviors occur when the mutual inclination between the inner and outer binaries is high.

In this study, we present two developments: (1) the perturbation potential in closed form up to the third order in a small parameter $\left(\alpha=a_{1} / a_{2}\right)$ when the perturbing star is put in elliptical and inclined orbits; in this case, the inner and outer orbits are mutually perturbed; (2) the perturbation potential in closed form expanded up to the fifth order in a small parameter $\left(\alpha=a_{1} / a_{2}\right)$, in the case where the perturbing star orbit is elliptical, planar, and fixed in space.

In the nomenclature used, $a_{1}$ is the semimajor axis of the planet and $a_{2}$ is the semimajor axis of the perturbing star. We then analyze the effects caused by the terms $P_{2}, P_{3}, P_{4}$, and $P_{5}$ (Legendre polynomial) in the orbital elements of the planet. The equations of motion are developed in closed forms to avoid expansions in power series of the eccentricity and inclination. We compared the models mentioned here in different orders and we also compared our results with the results obtained by Naoz et al. (2011, 2013). We performed several simulations to analyze the motion of the planet perturbed by the star. The approach used for the development of the equations is based on Brouwer (1959), Hori (1961), Prado (2003), and Yokoyama et al. (2003, 2008). Note that, in the literature, several authors have considered the three-body problem to study the dynamics of hierarchical triple systems, such as Krymolowski and Mazeh (1999), Ford et al. (2000, 2004), Takeda et al. (2008), Correia et al. (2011), Naoz et al. (2011), and Katz et al. (2011).

As a new aspect, in the present paper, the development of the equations of motion are explicitly presented up to the fifth order. We also show the importance of the $R_{4}$ and $R_{5}$ terms in changing the inversion times of the flip, i.e., the time where the inclination of the inner planet can flip from prograde to retrograde trajectories. We also show the dependence of the first flip with respect to the semimajor axis and eccentricity. In general, larger values of $e_{2}$ reduce the time required for the first inversion for low values of the semimajor axis of the perturbing star. There is a limit below which the inversion occurs and above which there is no more inversion (Fig. 6) for times up to $10^{7}$ years. As an example, for a semimajor axis of a perturbing star of $100 \mathrm{AU}$, an inversion only occurs for eccentricities equal to 0.5 and 0.6. Therefore, when considering distant stars, such as hierarchical triple systems, an inversion only occurs for large values of the eccentricity of the orbit of the perturbing star. The results show that the inclusion of the $R_{4}$ term gives results that are worse than those given by the $R_{3}$ term, but the inclusion of the $R_{5}$ term corrects and improves the results.

\section{Equations of motion}

The triple system under study is characterized by a planet $m_{1}$ which is orbiting a central star $m_{0}$ in an elliptical inner orbit around the center of mass of the system $m_{0}-m_{1}$. The star $m_{0}$ is also moving around the center of mass of the system $m_{0}-m_{1}$, and another perturbing star (brown dwarf- $m_{2}$ ) is moving in an outer elliptical orbit around the system's center of mass, but with a very distant trajectory that also has large eccentricity, as shown in Fig. 1. The vector $\mathbf{r}_{1}$ represents the position of $m_{1}$ with respect to $m_{0}$, and the vector $\mathbf{r}_{2}$ is the position 
Fig. 1 Scheme of coordinate system used to describe the triple star system (figure not to scale)

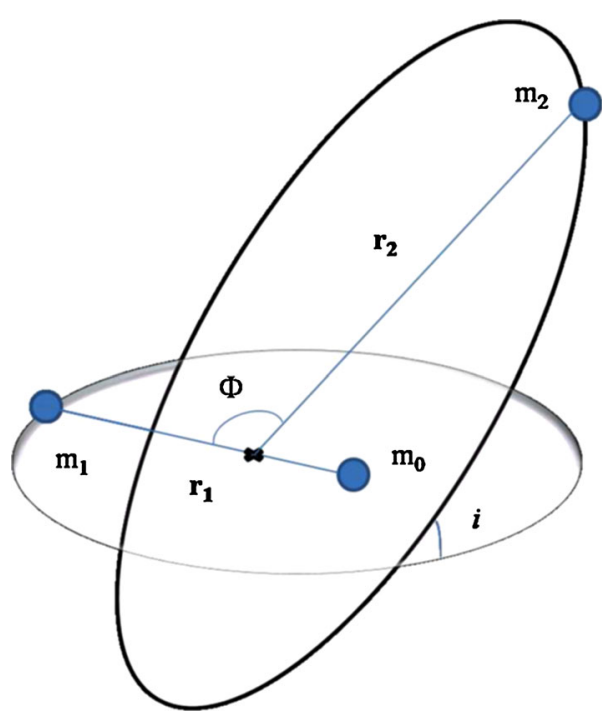

of the body $m_{2}$ with respect to the center of mass of the inner orbit. $\Phi$ is the angle between $\mathbf{r}_{1}$ and $\mathbf{r}_{2}$.

The Hamiltonian of the triple system can be written as follows (Harrington 1969; Ford et al. 2000, 2004; Takeda et al. 2008):

$$
\begin{aligned}
F= & \frac{G m_{0} m_{1}}{2 a_{1}}+\frac{G\left(m_{0}+m_{1}\right) m_{2}}{2 a_{2}} \\
& +\frac{G}{a_{2}} \sum_{j=2}^{\infty} \alpha^{j} M_{j}\left(\frac{r_{1}}{a_{1}}\right)^{j}\left(\frac{a_{2}}{r_{2}}\right)^{j+1} P_{j}(\cos \Phi),
\end{aligned}
$$

where $G$ is the gravitational constant, the $P_{j}$ are the Legendre polynomials, and

$$
M_{j}=m_{0} m_{1} m_{2} \frac{m_{0}^{j-1}-\left(-m_{1}\right)^{j-1}}{\left(m_{0}+m_{1}\right)^{j}} .
$$

To apply the secular approximation to real systems, several criteria need to be satisfied (e.g., the period of the outer orbit needs to be smaller than the Kozai time scales). Note that the system used in this paper can be modeled using average approximations, where we apply Eq. (25) of Naoz et al. (2013) to justify the averaging process (see also Antonini et al. 2014; Bode and Wegg 2013). In Naoz et al. (2013) the authors show that, considering only the quadrupole term in the potential, the inclination of the inner planet can flip from prograde to retrograde trajectories. The authors showed that several studies in the literature eliminated nodes incorrectly. To verify this assertion, we developed a perturbation potential taking into account the expression for $\cos \Phi$ written in the form (Yokoyama et al. 2003). 


$$
\begin{aligned}
\cos (\Phi)= & 1 / 4\left(-1+c_{2}\right)\left(-1+c_{1}\right) \cos \left(f_{1}+g_{1}-h_{1}-f_{2}-g_{2}+h_{2}\right) \\
& +1 / 4\left(1+c_{1}\right)\left(1+c_{2}\right) \cos \left(f_{1}+g_{1}+h_{1}-f_{2}-g_{2}-h_{2}\right) \\
& -1 / 4\left(1+c_{2}\right)\left(-1+c_{1}\right) \cos \left(f_{1}+g_{1}-h_{1}+f_{2}+g_{2}+h_{2}\right) \\
& -1 / 4\left(-1+c_{2}\right)\left(1+c_{1}\right) \cos \left(f_{1}+g_{1}+h_{1}+f_{2}+g_{2}-h_{2}\right) \\
& +1 / 2 s_{1} s_{2}\left(\cos \left(f_{1}+g_{1}-f_{2}-g_{2}\right)-\cos \left(f_{1}+g_{1}+f_{2}+g_{2}\right)\right)
\end{aligned}
$$

where we will use the shortcuts $s_{1}=\sin i_{1}, c_{1}=\cos i_{1}, s_{2}=\sin i_{2}$, and $c_{2}=\cos i_{2}$. Here $i_{j}, g_{j}, h_{j}$, and $f_{j}$ (for $j=1,2$ ) are the inclination, argument of the periastron, longitude of the ascending node, and true anomaly of the inner and outer orbits, respectively.

The perturbation potential was developed in closed form up to the third order in a small parameter $\left(\alpha=a_{1} / a_{2}\right)$, where the perturbing star is put in an elliptical inclined orbit. For the model considered in this paper, it is necessary to calculate the terms $R_{2}$ and $R_{3}$ of the perturbing function due to the $P_{2}$ and $P_{3}$ terms, respectively. We obtain

$$
\begin{aligned}
& R_{2}=\frac{G}{a_{2}} \alpha^{2} M_{2}\left(\frac{r_{1}}{a_{1}}\right)^{2}\left(\frac{a_{2}}{r_{2}}\right)^{3} P_{2}(\cos \Phi), \\
& R_{3}=\frac{G}{a_{2}} \alpha^{3} M_{3}\left(\frac{r_{1}}{a_{1}}\right)^{3}\left(\frac{a_{2}}{r_{2}}\right)^{4} P_{3}(\cos \Phi) .
\end{aligned}
$$

The perturbation potential given by Eq. (1) can be written as

$$
F=R_{0}+R_{2}+R_{3}
$$

where

$$
R_{0}=\frac{G m_{0} m_{1}}{2 a_{1}}+\frac{G\left(m_{0}+m_{1}\right) m_{2}}{2 a_{2}}
$$

To eliminate the short-period terms of the potential given by Eq. (6), the double-average method is applied with respect to the eccentric anomaly of the planet and of the true anomaly of the perturbing star. This is done using known equations from Celestial Mechanics:

$$
\begin{aligned}
\sin (f) & =\left(\sqrt{1-e^{2}} \sin (E)\right) /(1-e \cos (E)) ; \\
\cos (f) & =(\cos (E)-e) /(1-e \cos (E)), \\
r / a & =1-e \cos (E), \\
a / r & =(1+e \cos (f)) /\left(1-e^{2}\right), \\
\mathrm{d} l & =(1-e \cos (E)) \mathrm{d} E .
\end{aligned}
$$

We also used the area integral in the form (Brouwer 1959; Brouwer and Clemence 1961)

$$
\mathrm{d} l=\frac{1}{\sqrt{1-e^{2}}} \frac{r^{2}}{a^{2}} \mathrm{~d} f
$$

Using the expressions given by Eqs. (1)-(5), and taking into account the known relationships from the Celestial Mechanics mentioned previously, we integrate the equations with respect to the true and eccentric anomalies to eliminate short-period terms. Thus, we obtain the perturbation potential expanded up to the third order in a small parameter. The long-period perturbation potential $\left(R_{2}\right)$ can be written as 


$$
\begin{aligned}
R_{2 C}= & -\frac{45}{2} \frac{\beta_{3} L_{1}{ }^{4}}{L_{2}{ }^{6}\left(1-e_{2}\right)^{3 / 2}} \\
& \times\left(1 / 6 e_{1}^{2}\left(c_{2}-1\right)\left(c_{2}+1\right)\left(c_{1}+1\right)^{2} \cos \left(2 g_{1}-2 h_{2}+2 h_{1}\right)\right. \\
& +1 / 6 e_{1}^{2}\left(c_{2}-1\right)\left(c_{2}+1\right)\left(c_{1}-1\right)^{2} \cos \left(2 g_{1}+2 h_{2}-2 h_{1}\right) \\
& +2 / 3 c_{2} s_{1} s_{2} e_{1}^{2}\left(c_{1}-1\right) \cos \left(2 g_{1}-h_{1}+h_{2}\right) \\
& +2 / 3 c_{2} s_{1} s_{2} e_{1}^{2}\left(c_{1}+1\right) \cos \left(2 g_{1}+h_{1}-h_{2}\right) \\
& -1 / 5\left(c_{2}+1\right)\left(c_{1}-1\right)\left(c_{1}+1\right)\left(c_{2}-1\right)\left(2 / 3+e_{1}^{2}\right) \\
& \times \cos \left(-2 h_{2}+2 h_{1}\right)-4 / 5 c_{1} s_{2} s_{1}\left(2 / 3+e_{1}^{2}\right) c_{2} \\
& \times \cos \left(h_{1}-h_{2}\right)+\left(-1 / 3+c_{2}^{2}\right)\left(\left(c_{1}^{2}-1\right) e_{1}^{2} \cos \left(2 g_{1}\right)\right. \\
& \left.\left.-3 / 5\left(c_{1}^{2}-1 / 3\right)\left(2 / 3+e_{1}^{2}\right)\right)\right),
\end{aligned}
$$

where

$$
\begin{aligned}
L_{1} & =\frac{m_{0} m_{1} \sqrt{G\left(m_{0}+m_{1}\right) a_{1}}}{m_{0}+m_{1}}, \\
L_{2} & =\frac{m_{2}\left(m_{0}+m_{1}\right) \sqrt{G\left(m_{0}+m_{1}+m_{2}\right) a_{2}}}{m_{0}+m_{1}+m_{2}}, \\
\beta_{3} & =\frac{1}{16} \frac{G^{2}\left(m_{0}+m_{1}\right)^{7} m_{2}^{7}}{\left(m_{0}+m_{1}+m_{2}\right)^{3} m_{0}^{3} m_{1}^{3}}, \\
\beta_{4} & =\frac{1}{4} \frac{G^{2}\left(m_{0}+m_{1}\right)^{9} m_{2}{ }^{9}\left(m_{0}-m_{1}\right)}{\left(m_{0}+m_{1}+m_{2}\right)^{4} m_{0} m_{1}^{5}} .
\end{aligned}
$$

Note that the potential (three degrees of freedom) depends on the longitude of the ascending node of the inner $\left(h_{1}\right)$ and the outer $\left(h_{2}\right)$ orbits, but the number of degrees of freedom can be reduced after the equations of motion are derived (Naoz et al. 2013). Note also that the argument of periastron of the outer orbit does not appear in the second order of the potential. It is usually removed during the process of double averaging. Then we get $\mathrm{d} e_{2} / \mathrm{d} t=0$.

In Naoz et al. (2013) the authors show that, in the full three-body problem, it is incorrect to eliminate the nodes for the triple system. According to the authors, the $h_{1}-h_{2}=\pi$ term (often used in the literature) cannot be replaced at the Hamiltonian level. It is possible to use it only after deriving the equations of motion. In this way, they show that the mutual inclination can flip from prograde to retrograde trajectories when only the quadrupole term is taken into account.

The potential due to the $R_{3}$ term depends on the $g_{1}, g_{2}, h_{1}$, and $h_{2}$ terms (four degrees of freedom). The $R_{3}$ term was developed using Eq. (19), and $\cos (\Phi)$ was replaced by Eq. (3):

$$
R_{3}=\frac{2 \beta_{4} L_{1}{ }^{6} a_{2}{ }^{4} r_{1}{ }^{3}}{L_{2}{ }^{8} r_{2}{ }^{4} a_{1}{ }^{3}}\left(5 \cos ^{3}(\Phi)-3 \cos (\Phi)\right) .
$$

The perturbation potential due to the $R_{3}$ term is too long to show in the present paper, so we eliminate nodes before deriving the equations of motion so as to write a more compact equation. Note, however, that to perform the simulation, the equation $h_{1}-h_{2}=\pi$ was only used after the equations of motion were derived, as mentioned earlier. The perturbation potential due to the $R_{3}$ term is given by 


$$
\begin{aligned}
R_{3 C}= & -\frac{675}{128} \frac{e_{2} L_{1}{ }^{6} e_{1} \beta_{4}}{\left(-e_{2}{ }^{2}+1\right)^{5 / 2} L_{2}^{8}} \\
& \times\left(-\frac{7}{9} e_{1}^{2}\left(-c_{1} c_{2}+s_{1} s_{2}+1\right)\left(-c_{1} c_{2}+s_{1} s_{2}-1\right)^{2} \cos \left(3 g_{1}-g_{2}\right)\right. \\
& +\left(-c_{1} c_{2}+s_{1} s_{2}-1\right)\left(4 / 3+e_{1}^{2}\right)\left(c_{1}^{2} c_{2}^{2}+\left(-2 / 3-2 s_{1} s_{2}\right) c_{2} c_{1}+s_{1}^{2} s_{2}^{2}\right. \\
& \left.-1 / 15+2 / 3 s_{1} s_{2}\right) \cos \left(g_{1}-g_{2}\right)-\left(-c_{1} c_{2}+s_{1} s_{2}+1\right)\left(-\frac{7}{9} e_{1}^{2}\left(-c_{1} c_{2}\right.\right. \\
& \left.+s_{1} s_{2}+1\right)\left(-c_{1} c_{2}+s_{1} s_{2}-1\right) \cos \left(3 g_{1}+g_{2}\right)+\left(c_{1}{ }^{2} c_{2}{ }^{2}+\left(-2 s_{1} s_{2}\right.\right. \\
& \left.\left.\left.+2 / 3) c_{2} c_{1}+s_{1}{ }^{2} s_{2}{ }^{2}-1 / 15-2 / 3 s_{1} s_{2}\right)\left(4 / 3+e_{1}^{2}\right) \cos \left(g_{1}+g_{2}\right)\right)\right) .
\end{aligned}
$$

Our perturbation potential is written in the form

$$
R=R_{2 C}+R_{3 C} .
$$

Now using the $\cos \Phi$ term given by Kozai (1962), we get

$$
\cos \Phi=\cos \left(f_{1}+g_{1}\right) \cos \left(f_{2}+g_{2}\right)+c \sin \left(f_{1}+g_{1}\right) \sin \left(f_{2}+g_{2}\right),
$$

where we will use the shortcut $c=\cos i$. Here $i$ is the mutual inclination of the inner orbit with respect to the outer orbit.

The long-period perturbation potential ( $R_{2 K}$-quadrupole-Kozai) can be written as

$$
\begin{aligned}
R_{2 K}= & \frac{-\beta_{3} L_{1}^{4}}{L_{2}^{6}\left(1-e_{2}^{2}\right)^{3 / 2}} \times\left(15 e_{1}^{2} \cos \left(2 g_{1}\right) \cos ^{2}\left(i_{1}\right)\right. \\
& \left.-15 e_{1}^{2} \cos \left(2 g_{1}\right)-6 \cos ^{2}\left(i_{1}\right)-9 \cos ^{2}\left(i_{1}\right) e_{1}^{2}+2+3 e_{1}^{2}\right) .
\end{aligned}
$$

The potential depends on the argument of periastron of the inner orbit (one degree of freedom).

In Beaugé et al. (2012) is presented an approach to analyze the dynamical evolution of multiplanet systems due to their mutual gravitational interaction. The authors use the Hamiltonian system (quadrupole and octupole) that was derived from Laskar and Boué (2010). They analyze possible modes of motion for hierarchical (Valtonen and Karttunen 2006; Lee and Peale 2003), secular, or resonant configurations. The authors also present a review of the main techniques employed for the detection and orbital characterization of multiple-planet systems. To analyze the problem of three bodies (massive), in Correia et al. (2012) is considered the tidal effect due to the nonspherical shape of the planet $\left(J_{2}\right)$, coupled with the gravitational force due to the perturbing star.

Therefore, the long-period perturbation potential is written as

$$
\langle F\rangle=R_{0}+R_{2 C}+R_{3 C} .
$$

\subsection{Analyzing the $\boldsymbol{R}_{2}$ and $\boldsymbol{R}_{3}$ terms: perturbing star in elliptical and inclined orbit}

In this section we consider that the orbits of the planet and the perturbing star are mutually perturbed, so we have a system of nonlinear differential equations to integrate with eight equations of motion following application of the double-average method. In this case, the perturbation potential is considered up to the third order because the two orbits are mutually perturbed and the equations are very large, generating complex expansions if made up to the fifth order, with a large number of terms. In the next section the perturbing star will be considered fixed and planar, thereby reducing the previous number of differential equations by half, so in this case, the perturbation potential is presented up to the fifth order. 
Fig. $2 i_{1}$ versus $t$. Temporal evolution of inclination of planet. Initial conditions: $a_{1}=6 \mathrm{AU}$, $a_{2}=100 \mathrm{AU}, e_{1}=0.001$, $e_{2}=0.6, i_{1}=64.7^{\circ}, i_{2}=0.3^{\circ}$, $g_{1}=45^{\circ}, g_{2}=0^{\circ}$

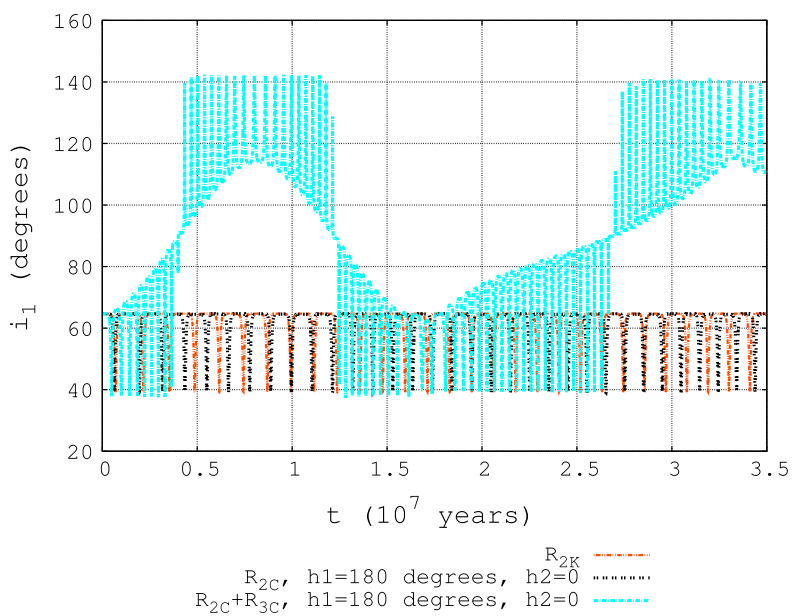

Here we replaced Eq. (24) in the Lagrange planetary equations (Kovalevsky 1967) and numerically integrated the set of nonlinear differential equations using the software Maple to analyze the orbital behavior of the planet for some particular cases.

A difference of the potential presented here with respect to the potential presented by Naoz et al. (2013) is that, for the $R_{2}$ and $R_{3}$ terms, we use Eq. (3) and elimination of the nodes was done following the derivation of the equations of motion, according to Naoz et al. (2013). In Naoz et al. (2013) the authors develop the $R_{2 N}$ term (quadrupole) using a different approach compared to that presented by Kozai (1962), where the relation $h_{1}-h_{2}=\pi$ was only used after deriving the equations of motion. This relation comes from the conservation of angular momentum. Another detail is that, in Naoz et al. (2013), the authors use the classical angle given by Kozai (Eq. 22) to develop the $R_{3 N}$ term (octupole). Here, the $R_{3 C}$ term was developed in the same way as was done for the term $R_{2 C}$, where we use Eq. (3), i.e., we do not eliminate the nodes before deriving the equations of motion for all orbital elements. Another difference is that, in the next section, we develop the perturbation potential up to the fifth order.

To investigate the effects of the $R_{2}$ and $R_{3}$ terms, we use the following initial conditions. The star has mass $1 M_{\odot}$, the planet has mass $1 M_{J}$, and the outer brown dwarf has mass $40 M_{J}$, where $M_{\odot}$ is the mass of the Sun and $1 M_{J}$ the mass of Jupiter. The inner orbit has $a_{1}=6 \mathrm{AU}$ and $e_{1}=0.001$, and the initial value for the relative inclination is $i=65^{\circ}$. These initial conditions were obtained from Naoz et al. (2011). Figure 2 shows a comparison between the perturbation potential models. Taking into account only the $R_{2}$ term, the red curve represents the potential when the nodes are eliminated in the Hamiltonian (Kozai 1962, $R_{2 K}$ ). The black curve shows the results when the nodes have not been eliminated before derivation of the equations of motion $\left(R_{2 C}\right)$. For this dynamical system, the inclination inversion phenomenon does not occur considering only the $R_{2 C}$ term. Note that, taking into account the potential given by Eq. (23), the inclination remains in a prograde orbit with constant amplitude (Fig. 2, red line) and, when considering the potential given by Eq. (14), the inclination also remains in a prograde orbit with the same constant amplitude, but the period of oscillation is different. Therefore, this shift in the period of oscillation is the practical result of the elimination of the nodes (Fig. 2, black line). Now, when the $R_{3 C}$ term is taken into account, the flip occurs between prograde and retrograde orbits (Fig. 2, blue line). When the $R_{3 C}$ term is taken into account, the argument of the periastron $\left(g_{2}\right)$ of the perturbing star appears in the equations 
Fig. $3 i_{2}$ versus $t$. Temporal evolution of inclination of perturbing star. Initial conditions: $a_{1}=6 \mathrm{AU}, a_{2}=100 \mathrm{AU}$, $e_{1}=0.001, e_{2}=0.6$, $i_{1}=64.7^{\circ}, i_{2}=0.3^{\circ}$, $g_{1}=45^{\circ}, g_{2}=0^{\circ}$

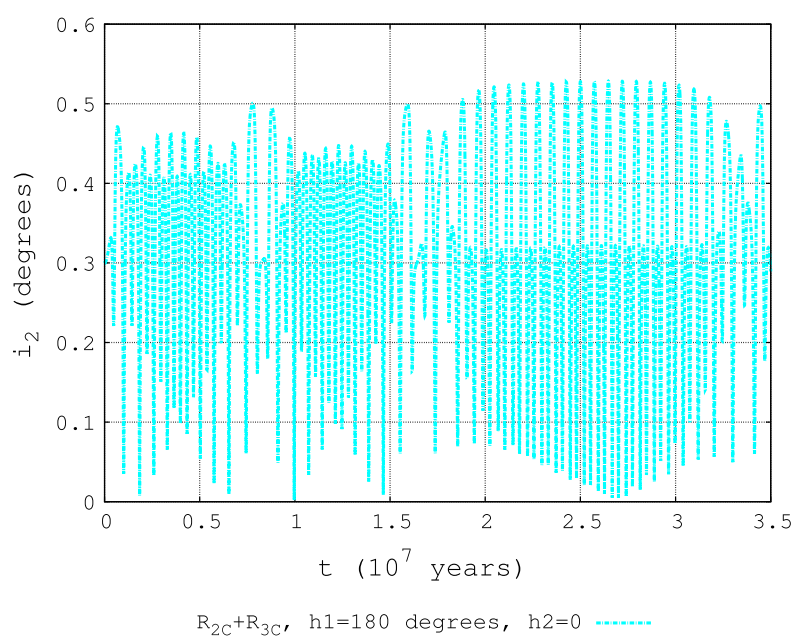

of motion, which contributes to the inversion time. In the second-order potential, this $\left(g_{2}\right)$ term does not appear in the equations of motion.

Figure 3 shows the inclination of the perturbing star with respect to the time. Note that the variation in the inclination is very small, i.e., the planet practically does not perturb the star. In the next section we show an approach where the perturbing star will be considered in the reference plane of the central star and in a fixed orbit in space. We will also develop the perturbation potential up to the fifth order in a small parameter $\left(\alpha=a_{1} / a_{2}\right)$.

We plot a diagram $g_{1}$ versus $e_{1}$ in Figs. 4 and 5. Note that in Figs. 4 and 5, the orbits librate around the equilibrium point for $g_{1}=90^{\circ}$ or $g_{1}=270^{\circ}$. Figure 4 shows the dynamics of orbits when only the $R_{2 C}$ term of the perturbation potential is taken into account and Fig. 5 considers the $R_{2 C}+R_{3 C}$ terms. Figure 4 shows orbits outlined by lines, whereas Fig. 5 shows orbits outlined by regions. This happens owing to the inclination, which flip between prograde and retrograde orbits.

Figure 6 shows the time required for the first inversion of the orbit on the vertical axis against the semimajor axis of the perturbing star on the horizontal axis. Figure 6 considers the longitude of the ascending node of the perturbing star to be $180^{\circ}$ and uses different values for $e_{2}$ and $a_{2}$. Some conclusions can be obtained from this figure. Regarding the difference in the dynamical models used, it is evident that, in general, larger values of $e_{2}$ reduce the time required for the first inversion for low values of the semimajor axis of the perturbing star.

In Fig. 6, eccentricities of $0.1-0.6$ for the perturbing star are considered. For $e_{2}=0.1$ and $e_{2}=0.2$ there was no inversion for the integration time used. For eccentricities above 0.2 , the occurrence of this phenomenon within a time span of $3.5 \times 10^{7}$ years depends on the semimajor axis of the perturbing star. There is a limit value below which the inversion occurs and above which no more inversions occur. When the eccentricity equals 0.3 , this limit is around $60 \mathrm{AU}$, when it equals 0.4 , the limit is near $90 \mathrm{AU}$, and if the eccentricity equals 0.5 , the limit is near $120 \mathrm{AU}$. Above this value an inversion always occurs in the range considered for the semimajor axis of the perturbing star $(150 \mathrm{AU})$. Figure 6 shows the exact locations of those inversions. Now considering values for $a_{2}$ up to $150 \mathrm{AU}$, only an eccentricity of 0.6 allows the phenomenon to occur, as seen in Fig. 6. This semimajor axis also has an expected strong effect on the inversion phenomenon since the closer the perturbing star is to the perturbed planet, the larger are the effects. This can be seen in Fig. 6 by looking at 
Fig. $4 e_{1}$ versus $g_{1}$. Time evolution for $3.5 \times 10^{7}$ years for eccentricity $e_{1}$ and argument of periastron $g_{1}$. Initial conditions: $a_{1}=6 \mathrm{AU}, a_{2}=100 \mathrm{AU}$, $e_{2}=0.6, i_{1}=64.7^{\circ}, i_{2}=0.3^{\circ}$, $g_{2}=0^{\circ}$. Perturbation potential $R_{2 C}$
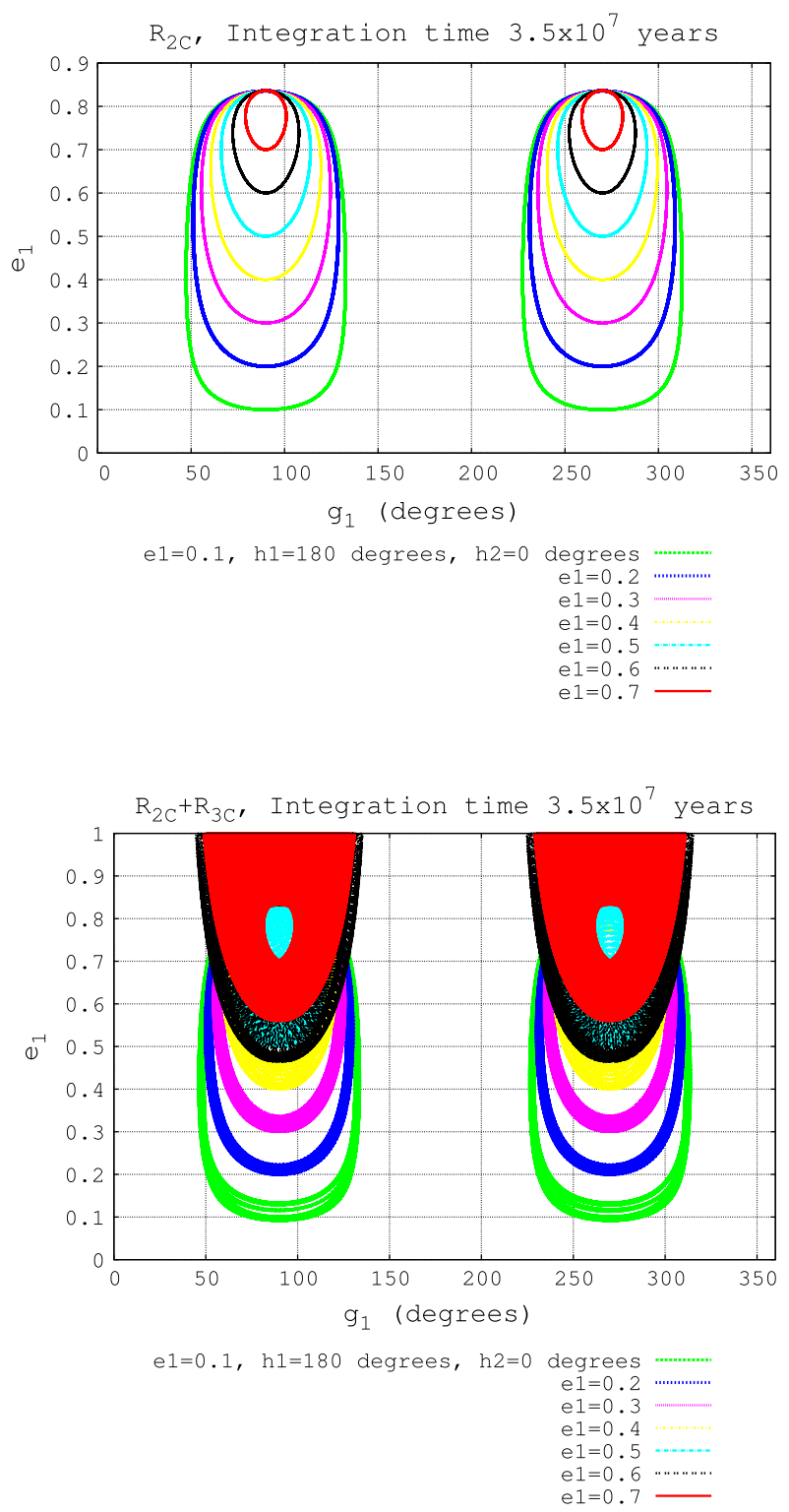

Fig. $5 e_{1}$ versus $g_{1}$. Time evolution for $3.5 \times 10^{7}$ years for eccentricity $e_{1}$ and argument of periastron $g_{1}$. Initial conditions: $a_{1}=6 \mathrm{AU}, a_{2}=100 \mathrm{AU}$, $e_{2}=0.6, i_{1}=64.7^{\circ}, i_{2}=0.3^{\circ}$, $g_{2}=0^{\circ}$. Perturbation potential $R_{2 C}+R_{3 C}$

the results for these values of the eccentricity. It is clear that the time for the first inversion increases with the semimajor axis. It is also evident that, in general, the value of the minimum eccentricity required for the phenomenon to occur decreases with increases in the semimajor axis. It is also clear that when the perturbing body is located in a position far from the planet (around $100 \mathrm{AU}$ ), inversion of the inclination only occurs for highly eccentric orbits of the perturbing star, for the time considered here for the simulations. Another fact that can be seen from Fig. 6 is that the time required for the first flip decreases with the eccentricity for a given value of the semimajor axis. The vertical lines in Fig. 6 make this clear. 
Fig. 6 Time of first flip of inclination versus $a_{2}$. Initial conditions: $a_{1}=6 \mathrm{AU}$, $e_{1}=0.001, i_{1}=64.7^{\circ}$, $i_{2}=0.3^{\circ}, g_{1}=45^{\circ}, g_{2}=0^{\circ}$

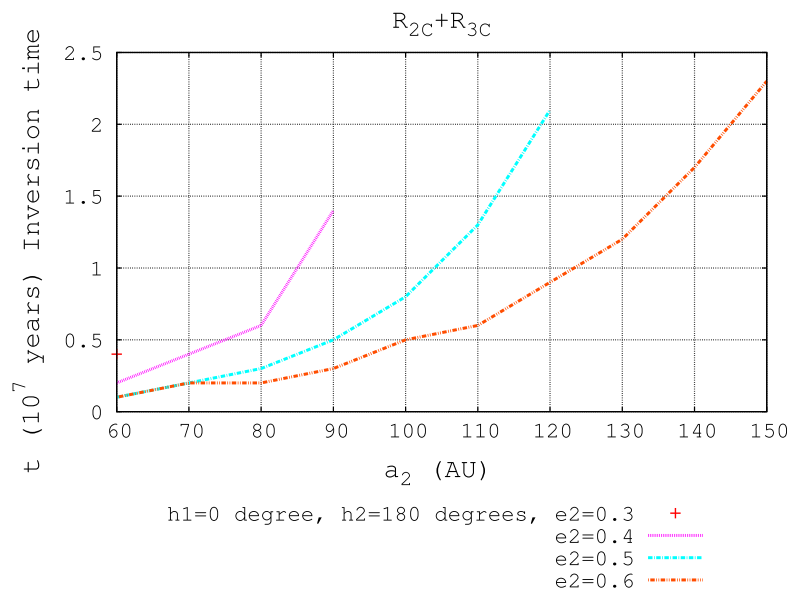

\section{Analyzing the $\boldsymbol{R}_{\mathbf{2}}, \boldsymbol{R}_{\mathbf{3}}, \boldsymbol{R}_{\mathbf{4}}$, and $\boldsymbol{R}_{\mathbf{5}}$ terms: perturbing star in planar orbit}

Now, let us consider the orbit of a perturbing body as planar and fixed in space. In Carvalho et al. (2013) the perturbation potential was developed up to the fourth order, but using Eq. (22) and the relation given by Lithwick and Naoz (2011) $\left(g_{2}=\pi-h_{1}\right)$. In other words, in addition to eliminating the nodes before deriving the equations of motion, as in the Kozai classical equation, the relation $g_{2}=\pi-h_{1}$ was also used to show the $h_{1}$ term explicitly in the equations of motion. Here, as was already shown, we use Eq. (3), given by Yokoyama et al. (2003), instead of Eq. (22), to avoid removing the nodes before deriving the equations of motion. However, in this section, the orbit of the perturbing star is planar and fixed in space. Then the orbital elements of the perturbing star are $i_{2}=0, g_{2}=0$, and $h_{2}=0$. Replacing these values in Eq. (3), we obtain the following equation:

$$
\begin{aligned}
\cos (\Phi)= & \frac{1}{2}\left(1+\cos \left(i_{1}\right)\right) \cos \left(f_{1}+g_{1}+h_{1}-f_{2}\right) \\
& +\frac{1}{2}\left(1-\cos \left(i_{1}\right)\right) \cos \left(f_{1}+g_{1}-h_{1}+f_{2}\right) .
\end{aligned}
$$

Those equations are written in an inertial reference system that has the equator of the main body in the $x-y$ plane.

For the model considered in this paper, it is necessary to calculate the terms $R_{2}$ to $R_{5}$ of the perturbing function because of the $P_{2}$ to $P_{5}$ terms, respectively. The results are as follows:

$$
\begin{aligned}
& R_{2}=\frac{G}{a_{2}} \alpha^{2} M_{2}\left(\frac{r_{1}}{a_{1}}\right)^{2}\left(\frac{a_{2}}{r_{2}}\right)^{3} P_{2}(\cos \Phi), \\
& R_{3}=\frac{G}{a_{2}} \alpha^{3} M_{3}\left(\frac{r_{1}}{a_{1}}\right)^{3}\left(\frac{a_{2}}{r_{2}}\right)^{4} P_{3}(\cos \Phi), \\
& R_{4}=\frac{G}{a_{2}} \alpha^{4} M_{4}\left(\frac{r_{1}}{a_{1}}\right)^{4}\left(\frac{a_{2}}{r_{2}}\right)^{5} P_{4}(\cos \Phi), \\
& R_{5}=\frac{G}{a_{2}} \alpha^{5} M_{5}\left(\frac{r_{1}}{a_{1}}\right)^{5}\left(\frac{a_{2}}{r_{2}}\right)^{6} P_{5}(\cos \Phi) .
\end{aligned}
$$


The perturbation potential given by Eq. (1) can be written as

$$
F=R_{0}+\sum_{j=2}^{5} R_{j C},
$$

where

$$
R_{0}=\frac{G m_{0} m_{1}}{2 a_{1}}+\frac{G\left(m_{0}+m_{1}\right) m_{2}}{2 a_{2}} .
$$

To eliminate the short-period terms of the potential given by Eq. (30), the double-average method is applied with respect to the eccentric anomaly of the planet and of the true anomaly of the perturbing star. Using the same approach as was used in the previous section but now assuming that the orbit of the perturbing star is planar and fixed in space, the equations of motion are now developed. Thus, we obtain the perturbation potential expanded up to the fifth order in a small parameter. The long-period perturbation potential can be written as

$$
\begin{aligned}
& R_{2 C}=15 \beta_{3} L_{1}^{4}\left(e_{1}^{2}\left(s_{1}^{2}\right) \cos \left(2 g_{1}\right)\right. \\
& \left.+3 / 5\left(e_{1}^{2}+2 / 3\right)\left(c_{1}^{2}-1 / 3\right)\right)\left(1-e_{2}{ }^{2}\right)^{-3 / 2} L_{2}{ }^{-6} \text {, } \\
& R_{3 C}=-\frac{675}{128} L_{1}{ }^{6} e_{2} \beta_{4} e_{1}\left(\frac{7}{9} e_{1}{ }^{2}\left(c_{1}+1\right)\left(c_{1}-1\right)^{2}\right. \\
& \times \cos \left(-h_{1}+3 g_{1}\right)-\left(c_{1}^{2}+2 / 3 c_{1}-1 / 15\right)\left(c_{1}-1\right) \\
& \times\left(e_{1}^{2}+4 / 3\right) \cos \left(g_{1}-h_{1}\right)+\left(c_{1}+1\right) \\
& \times\left(\left(-\frac{7}{9} e_{1}^{2} c_{1}^{2}+\frac{7}{9} e_{1}^{2}\right) \cos \left(h_{1}+3 g_{1}\right)\right. \\
& +\left(c_{1}^{2}-2 / 3 c_{1}-1 / 15\right) \cos \left(g_{1}+h_{1}\right) \\
& \left.\left.\times\left(e_{1}^{2}+4 / 3\right)\right)\right)\left(-e_{2}^{2}+1\right)^{-5 / 2} L_{2}^{-8} \text {, } \\
& R_{4 C}=\frac{19845}{1024} \beta_{5} L_{1}^{8} \\
& \times\left(4 / 9\left(e_{1}^{2}+2\right)\left(c_{1}^{2}+c_{1}+1 / 7\right) e_{2}^{2}\left(c_{1}-1\right)^{2} e_{1}^{2}\right. \\
& \times \cos \left(2 g_{1}-2 h_{1}\right)+4 / 9\left(e_{1}^{2}+2\right)\left(c_{1}+1\right)^{2} e_{2}^{2} \\
& \times\left(c_{1}^{2}-c_{1}+1 / 7\right) e_{1}^{2} \cos \left(2 g_{1}+2 h_{1}\right) \\
& -1 / 3 e_{1}^{4} e_{2}^{2}\left(c_{1}+1\right)\left(c_{1}-1\right)^{3} \cos \left(4 g_{1}-2 h_{1}\right) \\
& -1 / 3 e_{1}^{4} e_{2}^{2}\left(c_{1}-1\right)\left(c_{1}+1\right)^{3} \cos \left(4 g_{1}+2 h_{1}\right) \\
& -4 / 3\left(e_{1}^{2}+2\right)\left(c_{1}+1\right)\left(c_{1}^{2}-1 / 7\right)\left(e_{2}^{2}+2 / 3\right) \\
& \times\left(c_{1}-1\right) e_{1}^{2} \cos \left(2 g_{1}\right)-\frac{10}{21}\left(c_{1}+1\right) e_{2}^{2} \\
& \times\left(e_{1}^{4}+8 / 3 e_{1}^{2}+\frac{8}{15}\right)\left(c_{1}^{2}-1 / 7\right)\left(c_{1}-1\right) \cos \left(2 h_{1}\right) \\
& +\left(e_{2}^{2}+2 / 3\right)\left(e_{1}^{4}\left(c_{1}-1\right)^{2}\left(c_{1}+1\right)^{2} \cos \left(4 g_{1}\right)\right. \\
& \left.\left.+5 / 7\left(e_{1}^{4}+8 / 3 e_{1}^{2}+\frac{8}{15}\right)\left(c_{1}^{4}-6 / 7 c_{1}^{2}+\frac{3}{35}\right)\right)\right) \\
& \times\left(-e_{2}^{2}+1\right)^{-7 / 2} L_{2}{ }^{-10} \text {, }
\end{aligned}
$$




$$
\begin{aligned}
& R_{5 C}=\frac{165375}{4096} \beta_{6} L_{1}^{10} \\
& \times e_{1}\left(-\frac{9}{10}\left(c_{1}-1\right)^{2} e_{1}^{2}\left(e_{1}^{2}+8 / 3\right)\left(c_{1}+1\right)\left(e_{2}^{2}+4 / 3\right)\right. \\
& \times\left(c_{1}^{2}+2 / 5 c_{1}-1 / 15\right) \cos \left(-h_{1}+3 g_{1}\right)+\frac{3}{20}\left(c_{1}-1\right)^{3}\left(c_{1}+\frac{13}{15}\right) e_{1}^{2} \\
& \times\left(e_{1}^{2}+8 / 3\right)\left(c_{1}+1 / 3\right) e_{2}^{2} \cos \left(3 g_{1}-3 h_{1}\right)-\frac{3}{20}\left(c_{1}-1 / 3\right) e_{1}^{2} \\
& \times\left(e_{1}^{2}+8 / 3\right)\left(c_{1}+1\right)^{3} e_{2}^{2}\left(c_{1}-\frac{13}{15}\right) \cos \left(3 g_{1}+3 h_{1}\right)+\frac{33}{50}\left(c_{1}-1\right)^{3} e_{1}^{4} \\
& \times\left(c_{1}+1\right)^{2}\left(e_{2}^{2}+4 / 3\right) \cos \left(5 g_{1}-h_{1}\right)-\frac{11}{100} e_{1}^{4} e_{2}^{2}\left(c_{1}+1\right) \\
& \times\left(c_{1}-1\right)^{4} \cos \left(5 g_{1}-3 h_{1}\right)+\frac{11}{100} e_{1}{ }^{4} e_{2}^{2}\left(c_{1}-1\right)\left(c_{1}+1\right)^{4} \cos \left(5 g_{1}+3 h_{1}\right) \\
& +\left(c_{1}-1\right)\left(c_{1}{ }^{4}+4 / 5 c_{1}{ }^{3}-2 / 5 c_{1}{ }^{2}-\frac{4}{15} c_{1}+\frac{1}{105}\right)\left(e_{2}^{2}+4 / 3\right) \\
& \times\left(e_{1}^{4}+4 e_{1}^{2}+8 / 5\right) \cos \left(g_{1}-h_{1}\right)-\left(-\frac{9}{10}\left(c_{1}-1\right) e_{1}^{2}\left(e_{1}^{2}+8 / 3\right)\right. \\
& \times\left(c_{1}+1\right)\left(c_{1}^{2}-2 / 5 c_{1}-1 / 15\right)\left(e_{2}^{2}+4 / 3\right) \cos \left(h_{1}+3 g_{1}\right) \\
& +1 / 6\left(c_{1}-1\right)^{2} e_{2}^{2}\left(e_{1}^{4}+4 e_{1}^{2}+8 / 5\right)\left(c_{1}^{2}+2 / 5 c_{1}-1 / 15\right) \cos \left(g_{1}-3 h_{1}\right) \\
& -1 / 6\left(c_{1}-1\right)\left(c_{1}+1\right)\left(c_{1}^{2}-2 / 5 c_{1}-1 / 15\right) e_{2}{ }^{2}\left(e_{1}^{4}+4 e_{1}{ }^{2}+8 / 5\right) \\
& \times \cos \left(g_{1}+3 h_{1}\right)+\left(\frac{33}{50} e_{1}^{4}\left(c_{1}-1\right)^{2}\left(c_{1}+1\right)^{2} \cos \left(5 g_{1}+h_{1}\right)\right. \\
& \left.+\cos \left(g_{1}+h_{1}\right)\left(c_{1}^{4}-4 / 5 c_{1}^{3}-2 / 5 c_{1}^{2}+\frac{4}{15} c_{1}+\frac{1}{105}\right)\left(e_{1}^{4}+4 e_{1}^{2}+8 / 5\right)\right) \\
& \left.\left.\left.\times\left(e_{2}^{2}+4 / 3\right)\right)\left(c_{1}+1\right)\right) e_{2}\right) \\
& \times\left(-e_{2}{ }^{2}+1\right)^{-9 / 2} L_{2}{ }^{-12} \text {, }
\end{aligned}
$$

where we use the shortcut $s_{1}=\sin i_{1}, c_{1}=\cos i_{1}$, and where

$$
\begin{aligned}
& \beta_{5}=\frac{1}{8} \frac{G^{2}\left(m_{0}+m_{1}\right)^{10} m_{2}{ }^{11}\left(m_{0}{ }^{3}+m_{1}^{3}\right)}{\left(m_{0}+m_{1}+m_{2}\right)^{5} m_{0} m_{1} m^{7}}, \\
& \beta_{6}=\frac{1}{8} \frac{G^{2}\left(m_{0}+m_{1}\right)^{12} m_{2}{ }^{13}\left(m_{0}{ }^{4}-m_{1}{ }^{4}\right)}{\left(m_{0}+m_{1}+m_{2}\right)^{6} m_{0}{ }^{9} m_{1}{ }^{9}} .
\end{aligned}
$$

Therefore, the long-period perturbation potential is written as

$$
\langle F\rangle=\sum_{j=2}^{5} R_{j C} .
$$

It is possible replaced Eq. (38) in the Lagrange planetary equations (Kovalevsky 1967) to analyze the evolution of the orbital elements of the planet, in particular the inclination and eccentricity. The evolution of those elements can be obtained from numerical simulations, which are performed using the Maple software. 
Fig. 7 Temporal evolution of inclination. Here "(pe)" means that the inner and outer orbits are mutually perturbed, and "(fix)" means that the orbit of the perturbing star is fixed in space. Initial conditions: $a_{1}=6 \mathrm{AU}$, $e_{1}=0.01, a_{2}=100 \mathrm{AU}$, $e_{2}=0.6, i_{1}=65^{\circ}, g_{1}=0^{\circ}$. The star has mass $1 M_{\odot}$, the planet has mass $1 M_{J}$, and the outer brown dwarf has mass $40 M_{J}$
Fig. 8 Temporal evolution of inclination. Here "(pe)" means that the inner and outer orbits are perturbed mutually, and "(fix)" means that the orbit of the perturbing star is fixed in space. Initial conditions: $a_{1}=6 \mathrm{AU}$, $e_{1}=0.01, a_{2}=100 \mathrm{AU}$, $e_{2}=0.6, i_{1}=65^{\circ}$ and $g_{1}=0^{\circ}$. The star has mass $1 M_{\odot}$, the planet has mass $1 M_{J}$, and the outer brown dwarf has mass $40 M_{J}$
Temporal evolution of the inclination
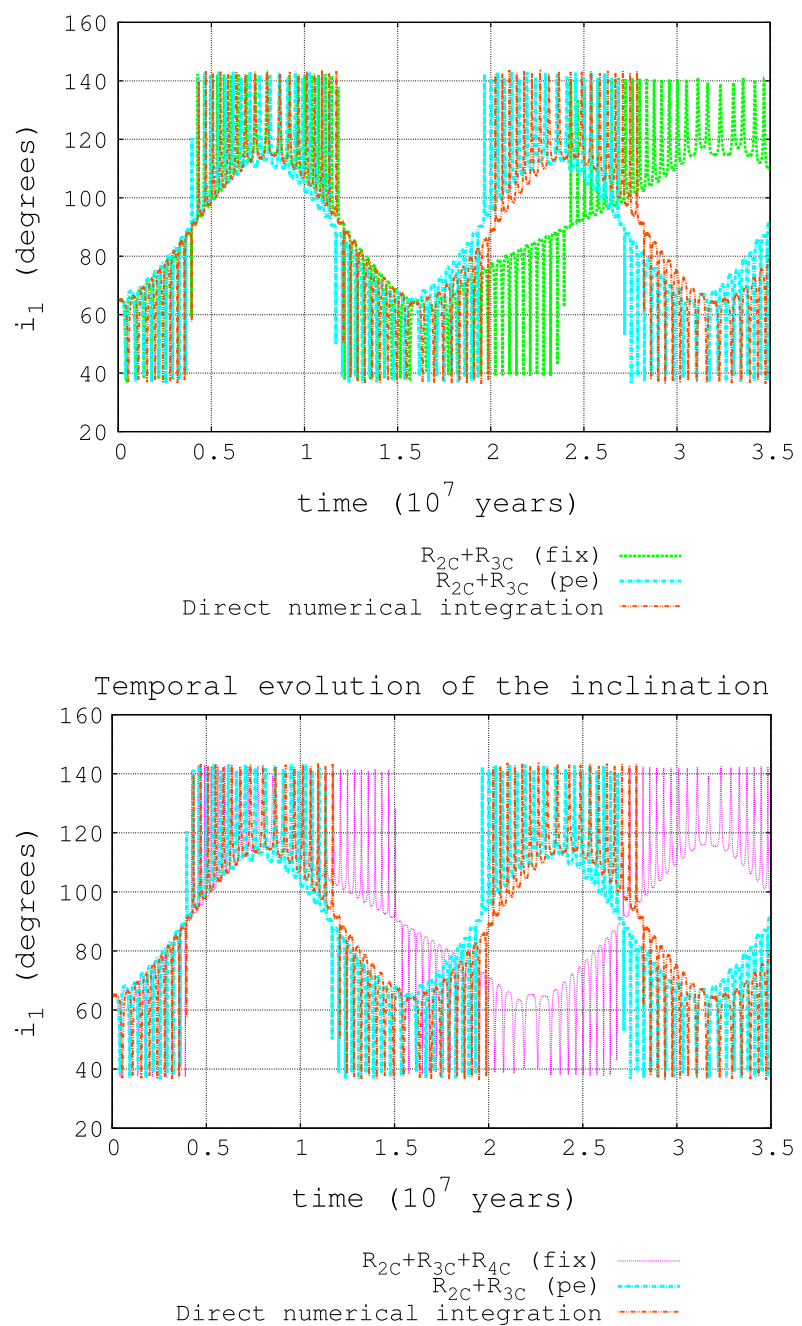

The perturbation potential given by Eq. (38) is now replaced in the Lagrange planetary equations and numerically integrated. Figures 7, 8, and 9 show the effects of the perturbation potential on the planet's orbit for different orders of the perturbation potential. We also performed full numerical integrations using the Bulirsch-Stoer method available in the Mercury package (Chambers 1999). The red curve (Figs. 7, 8, and 9) was generated from the direct numerical integration of the three-body problem. The blue curve represents the dynamics of the planet when the inner and outer orbits are mutually perturbed [Eq. 24, $R_{2 C}+R_{3 C}$ (pe)]. Note that when the mutual perturbation of the two orbits is taken into account, even observing that the perturbing star is much less perturbed than the planet's orbit (Fig. 3), the result is in agreement with the numerical simulation. But when only the perturbation of the inner orbit is considered and the $R_{2 C}+R_{3 C}$ (fix) terms in the perturbation potential are taken into account, i.e., the orbit of the perturbing star is considered fixed and planar, the planet inclination behavior differs, in terms of the variation of the inversion time, from the numerical 
Fig. 9 Temporal evolution of inclination. Here "(pe)" means that the inner and outer orbits are perturbed mutually, and "(fix)" means that the orbit of the perturbing star is fixed in space. Initial conditions: $a_{1}=6 \mathrm{AU}$, $e_{1}=0.01, a_{2}=100 \mathrm{AU}$, $e_{2}=0.6, i_{1}=65^{\circ}, g_{1}=0^{\circ}$. The star has mass $1 M_{\odot}$, the planet has mass $1 M_{J}$, and the outer brown dwarf has mass $40 M_{J}$

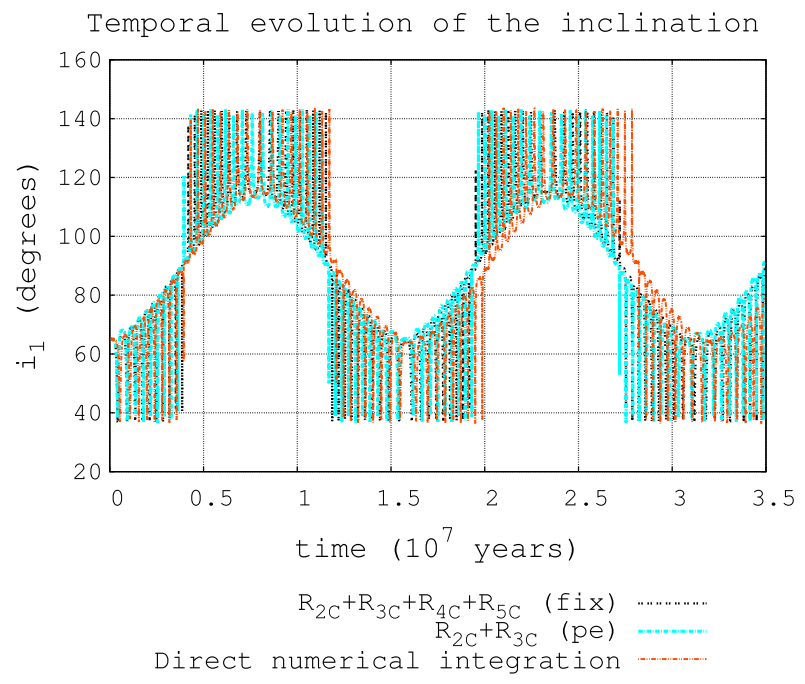

result after the third inversion. From that moment the results are completely different from the numerical simulation. Now, when considering up to the fourth order of the perturbation potential [ $\sum_{j=2}^{4} R_{j C}(\mathrm{fix})$ ], the inclination behavior differs, also in terms of the variation of the inversion time, from the numerical results as early as the second inversion. When considering up to the fourth order of the perturbation potential, the inclination characteristic of the planet diverges from the numerical results, namely, the term $R_{4 C}$ (fix) contributes to an increase in the inversion time of the inclination and thus the orbit remains for a longer time on a prograde or retrograde trajectory. The results, considering the perturbation potential expanded up to the fifth order $\left[\sum_{j=2}^{5} R_{j} C\right.$ (fix)], show that they are in agreement with the numerical simulations and in agreement with the case when the octupole term is taken account, where the two orbits are mutually perturbed and inclined. When the perturbation potential up to the fifth order is taken into account, the planet's inclination behavior shows a slight difference from the result of direct simulations. These differences start to appear in the third inversion, but this difference is very small compared to those presented by the perturbation potential developed up to the third or fourth order. Thus, one realizes that the term $R_{5 C}$ (fix) contributes to the results of the equations developed here and it can reproduce more faithfully the results obtained by the direct numerical integration of the three-body problem. In this way, Fig. 7 shows the results under the assumption that the star remains in a fixed orbit.

We note that the octupole term is strongly dominant in the inversion inclination phenomenon, in particular in the period where the inclination migrates from prograde to retrograde orbits. If the system analyzed considers that the two orbits are mutually perturbed, the dynamics is well represented considering up to the third order of the perturbation potential. However, when the external orbit is planar and fixed, it is necessary to expand the potential up to the fifth order, as shown in Figs. 7, 8, and 9, where the lower-order terms do not represent the dynamical behavior compared to the direct numerical integration of the three-body problem. Note also that the terms of odd order of the expansion in Legendre polynomials are strongly dominant with respect to the terms of even order. Figure 10 shows the behavior of the planet's orbit for different initial inclinations. In all cases analyzed on which the inclination inversion phenomenon occurs, what changes is only the time that each orbit requires to migrate from 
Fig. 10 Temporal evolution of inclination. Initial conditions: $a_{1}=6 \mathrm{AU}, a_{2}=100 \mathrm{AU}$, $e_{1}=0.01, e_{2}=0.6, g_{1}=0$, $h_{1}=180^{\circ}$. The star has mass $1 M_{\odot}$, the planet has mass $1 M_{J}$, and the outer brown dwarf has mass $40 M_{J}$

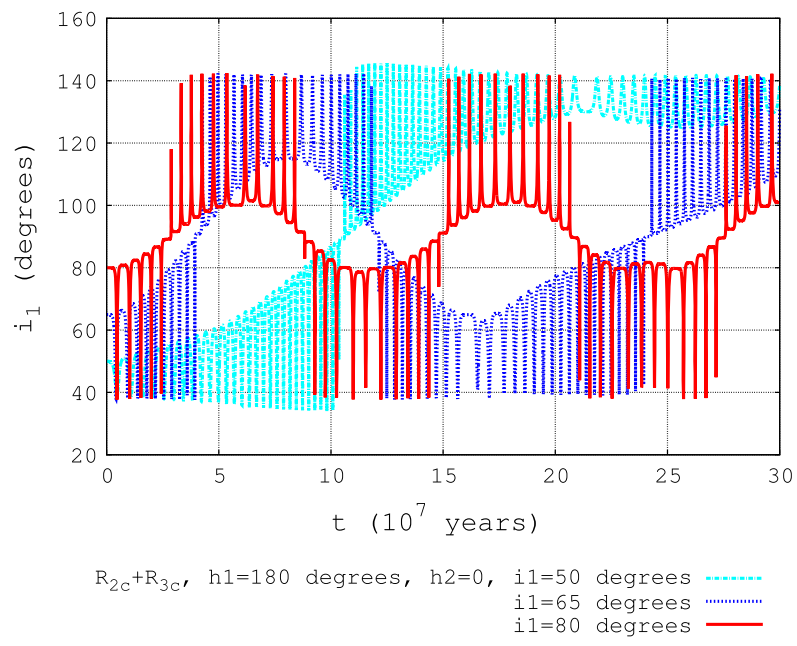

Fig. 11 Temporal evolution of eccentricity $\left(1-e_{1}\right) \times \mathrm{t}$. Direct numerical integration of problem of three bodies. Initial conditions: $a_{1}=6 \mathrm{AU}, a_{2}=100 \mathrm{AU}$, $e_{1}=0.01, e_{2}=0.6, i_{1}=65^{\circ}$, $g_{1}=0, h_{1}=180^{\circ}$. The star has mass $1 M_{\odot}$, the planet has mass $1 M_{J}$, and the outer brown dwarf has mass $40 M_{J}$

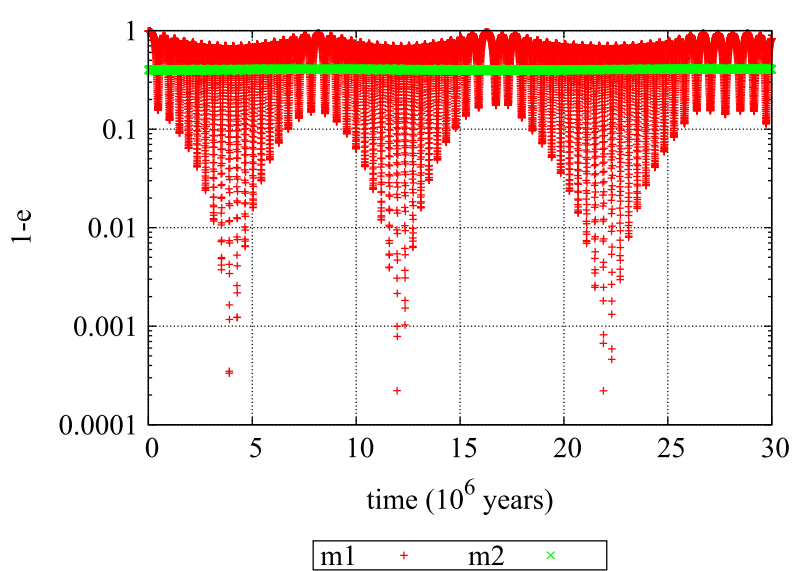

prograde to retrograde. Note that, as expected, the higher the inclination, the shorter the time required for the flip. Although expected, Fig. 10 quantifies these results, showing exactly the time required for the flip for each initial inclination. Figure 11 and the red curves shown in Figs. 7, 8, and 9 were generated from the direct numerical integration of the three-body problem. Figure 11 shows that the eccentricity can reach very high values and their inclination can become higher than $90^{\circ}$, when terms of higher orders are included in the perturbation potential, as shown in Figs. 7, 8, and 9.

Figures 12, 13, and 14 show the characteristic of a highly eccentric orbit $\left(e_{1}=0.9\right)$ and low inclination $\left(i_{1}=5^{\circ}\right)$. The initial conditions were obtained from Li et al. (2014a). Note that considering a planet with highly eccentric orbit and low inclination, the dynamical behavior of the inclination is totally different compared to the previously analyzed cases. Here, after the flip, the inclination oscillates for a while around $180^{\circ}$. The red line was generated from the direct numerical integration of the three-body problem. Note that the test particle exhibits an almost $180^{\circ}$ flip in a coplanar configuration. Note that, in this case, considering the perturbation potential expanded up to the fourth order, the inclination behavior does not diverge from the numerical result, as in the case presented in Fig. 8. The curve that is 
Fig. 12 Temporal evolution of inclination. Initial conditions: $a_{1}=1 \mathrm{AU}, a_{2}=50 \mathrm{AU}$, $e_{1}=0.9, e_{2}=0.7, i_{1}=5^{\circ}$, $g_{1}=0, h_{1}=180^{\circ}$. The star has mass $1 M_{\odot}$, the planet has mass $10^{-3} M_{\odot}$, and the outer brown dwarf has mass $0.02 M_{\odot}$

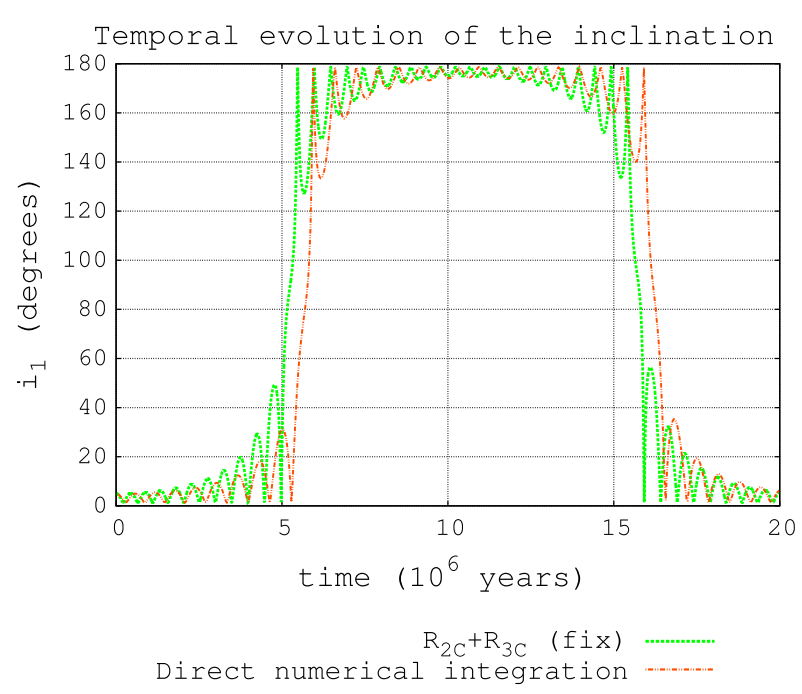

Fig. 13 Temporal evolution of inclination. Initial conditions: $a_{1}=1 \mathrm{AU}, a_{2}=50 \mathrm{AU}$, $e_{1}=0.9, e_{2}=0.7, i_{1}=5^{\circ}$, $g_{1}=0, h_{1}=180^{\circ}$. The star has mass $1 M_{\odot}$, the planet has mass $10^{-3} M_{\odot}$, and the outer brown dwarf has mass $0.02 M_{\odot}$

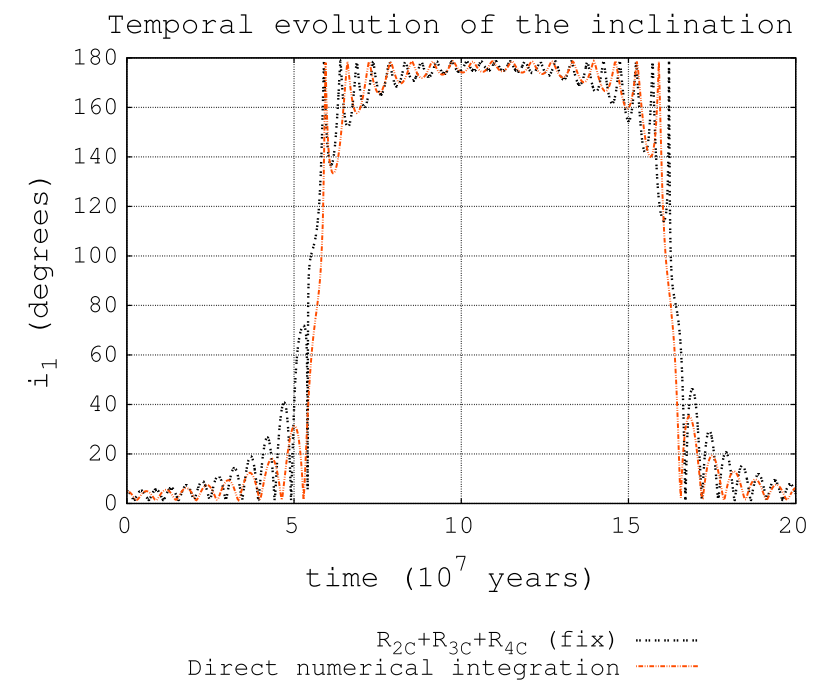

closer to the one that represents the direct numerical integration is the one that takes into account the potential expanded up to the fourth order (Fig. 13). Note that the potential that has expanded up to the fifth order approaches the numerical result. The second best model is the one that considers the potential that has expanded up to the third order (Figs. 12 and 14). Another difference shown in the dynamics is that, looking at Figs. 7, 8, and 9, we see that the inclination migrates smoothly from prograde to retrograde orbits, while in the cases of Figs. 12, 13, and 14 this transition is not smooth but changes abruptly in the inclination.

Comparing Figs. 12, 13, and 14 with Fig. 2, from Li et al. (2014b) we observe that there is a difference between the models. In our case a prograde orbit migrates to retrograde in a time frame of around $5 \times 10^{6}$ years, while in Fig. 2, from Li et al. (2014b), the migration occurs in a time frame of around $10 \times 10^{6}$ years. 
Fig. 14 Temporal evolution of inclination. Initial conditions: $a_{1}=1 \mathrm{AU}, a_{2}=50 \mathrm{AU}$, $e_{1}=0.9, e_{2}=0.7, i_{1}=5^{\circ}$, $g_{1}=0, h_{1}=180^{\circ}$. The star has mass $1 M_{\odot}$, the planet has mass $10^{-3} M_{\odot}$, and the outer brown dwarf has mass $0.02 M_{\odot}$

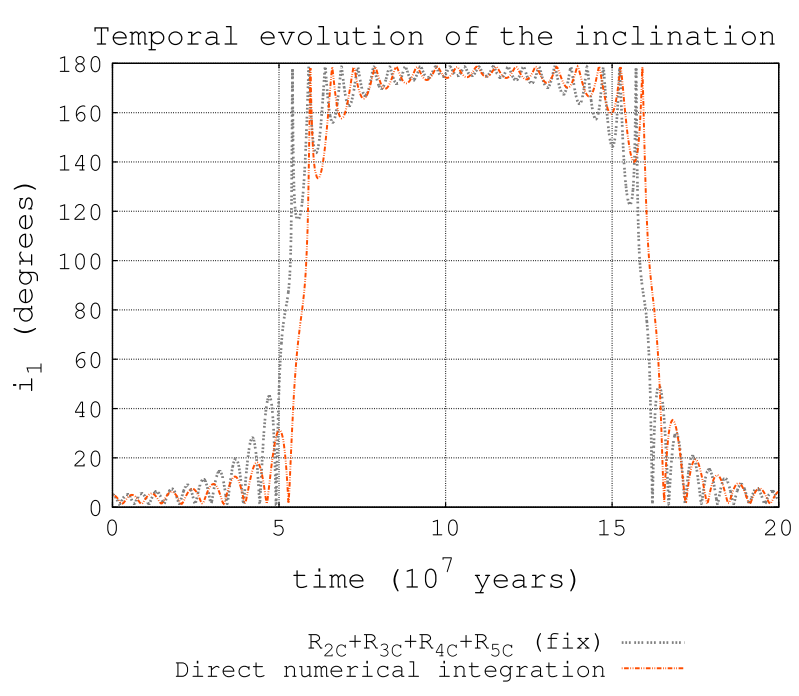

In Li et al. (2014b), the authors show an analytic equation for the flip criterion for the coplanar case (i.e., values of $i_{1}$ near zero). Here we use Eq. (14), as shown in Li et al. (2014b), to verify the initial conditions used in Figs. 12, 13, and 14. Performing the calculations, we find that the set of initial conditions satisfies the flip criterion defined by Eq. (14). Using the same initial conditions, but changing the value of the eccentricity of the planet, we found that Eq. (14) remains satisfied for $e_{1} \geq 0.46$. Now, considering the initial conditions used in other figures in the paper, with $i_{1} \gg 0$, Eq. (14) from Li et al. (2014b) cannot be used since it was defined for the coplanar case.

Figure 15 shows the inversion times for different values of the semimajor axis of the planet and of the perturbing star. The semimajor axis of the planet is considered as ranging from 1 to $10 \mathrm{AU}$, and the semimajor axis of the star ranges from 60 to $150 \mathrm{AU}$. Note that the inversion only occurs for the integration time used $\left(3.5 \times 10^{7}\right.$ years $)$ from $a_{1}=3 \mathrm{AU}$. For $a_{1}=3 \mathrm{AU}$, the inversion occurs only for the following values of $a_{2}: 60,70$, and $80 \mathrm{AU}$. For values of $a_{1}$ from $6 \mathrm{AU}$ the inversion occurs for all values of $a_{2}$ considered. Note that when the perturbing star is near the planet, the inversion occurs very fast, when compared with situations where the perturbing star is located in a position farther from the planet. This is expected because the disturbance decreases with the inverse square of the distance. The diagram shown in Fig. 15 gives the times for the first inversion considering in the perturbation potential the $\sum_{j=2}^{4} R_{j C}$ terms. Comparing Fig. 15 with Fig. 16, we observe that, in general, the inversion time is reduced when a potential that has expanded up to the fifth order is taken into account.

To verify the differences between $t_{\text {inv }}$ considering the perturbation potential with and without the $R_{4 C}$ and $R_{5 C}$ terms, we performed numerical integrations for several ranges of $e_{1}$ and $e_{2}$. Figure 17 presents the flip times obtained for the case with a perturbation potential given by $R_{2 C}+R_{3 C}$. As expected, $t_{\text {inv }}$ decreases with increases in the eccentricities $\left(e_{1}\right.$ and $e_{2}$ ). Note that (Fig. 17) for high values of the perturbing star eccentricity $\left(e_{2}=0.5,0.6\right.$, and 0.7 ) there is always inversion in the inclination and the flip occurs in shorter times. The distance and eccentricity of the perturbing star strongly influence the time of inversion of the planet's inclination.

The modulus of the difference between the flip times considering the perturbation potential $R_{2 C}+R_{3 C}+R_{4 C}$ and the perturbation potential $R_{2 C}+R_{3 C}$ is shown in Fig. 18. This 
Fig. $15 t_{\text {inv }} \times a_{2}$. Initial conditions: $e_{1}=0.01, e_{2}=0.6$, $i_{1}=65^{\circ}, g_{1}=0, h_{1}=180^{\circ}$.

The star has mass $1 M_{\odot}$, the planet has mass $1 M_{J}$, and the outer brown dwarf has mass $40 M_{J}$. Potential expanded up to fourth order $\left(\sum_{j=2}^{4} R_{j C}\right)$
Fig. $16 t_{\text {inv }} \times a_{2}$. Initial conditions: $e_{1}=0.01, e_{2}=0.6$, $i_{1}=65^{\circ}, g_{1}=0, h_{1}=180^{\circ}$. The star has mass $1 M_{\odot}$, the planet has mass $1 M_{J}$, and the outer brown dwarf has mass $40 M_{J}$. Potential expanded up to the fifth order $\left(\sum_{j=2}^{5} R_{j C}\right)$
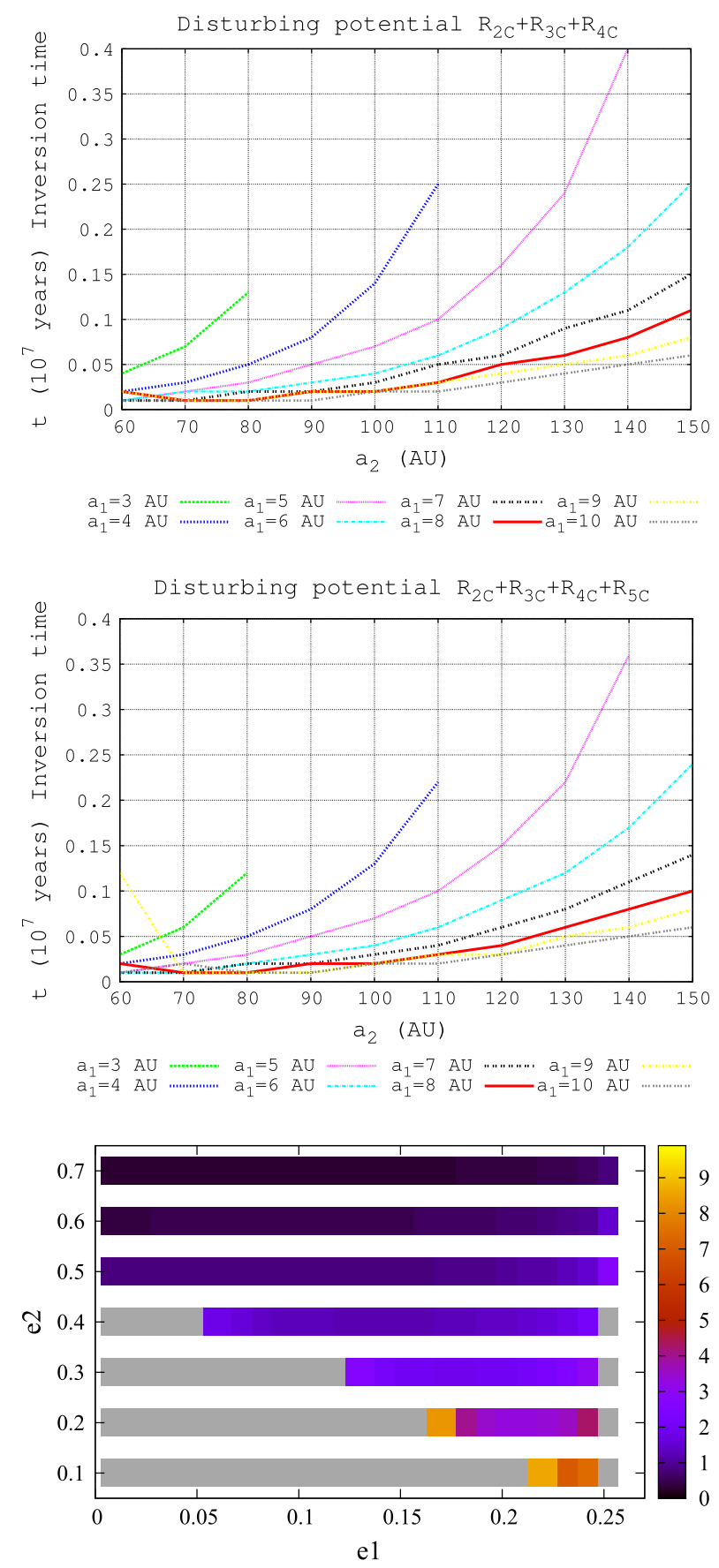

Fig. $17 e_{2} \times e_{1} \times t_{\text {inv }}$.

Perturbation potential

$R_{2 C}+R_{3 C}$. Initial conditions: $a_{1}=6 \mathrm{AU}, a_{2}=100 \mathrm{AU}$, $i_{1}=65^{\circ}, g_{1}=0^{\circ}, h_{1}=180^{\circ}$. The star has mass $1 M_{\odot}$, the planet has mass $1 M_{J}$, and the outer brown dwarf has mass $40 M_{J}$; colors: time in multiples of $10^{7}$ years; gray indicates that no flip occurred during integration

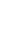

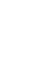

(n)

figure shows the effect of the $R_{4}$ term. The modulus of the difference between the flip times considering the perturbation potential $R_{2 C}+R_{3 C}+R_{4 C}+R_{5}$ and the potential $R_{2 C}+R_{3 C}$ is shown in Fig. 19. This figure shows the effect of the $R_{4}+R_{5}$ terms. Comparing Figs. 18 and 19 
Fig. $18 e_{2} \times e_{1} \times t_{\text {inv }}$.

Difference between inversion times using $R_{2 C}+R_{3 C}+R_{4 C}$ and using only $R_{2 C}+R_{3 C}$. Initial conditions: $a_{1}=6 \mathrm{AU}$, $a_{2}=100 \mathrm{AU}, i_{1}=65^{\circ}$, $g_{1}=0^{\circ}, h_{1}=180^{\circ}$. The star has mass $1 M_{\odot}$, the planet has mass $1 M_{J}$, and the outer brown dwarf has mass $40 M_{J}$; colors: time in multiples of $10^{7}$ years; gray indicates that no flip occurred during integration

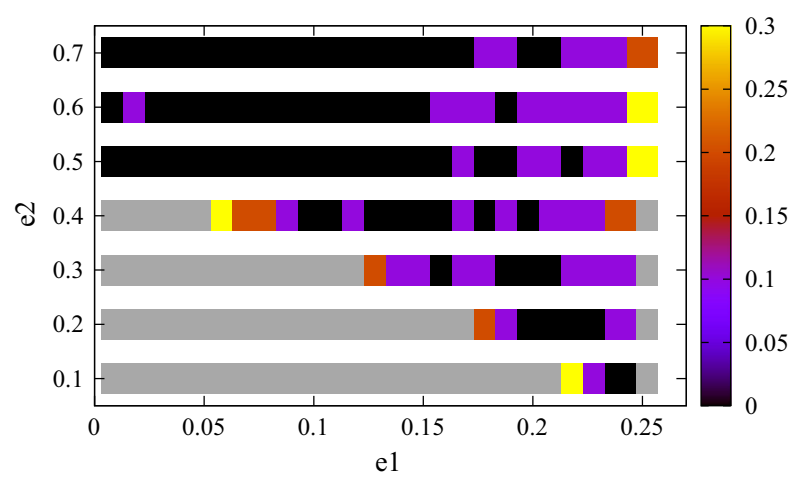

Fig. $19 e_{2} \times e_{1} \times t_{\text {inv }}$. Difference between inversion times using $R_{2 C}+R_{3 C}+$ $R_{4 C}+R_{5 C}$ and using only $R_{2 C}+R_{3 C}$. Initial conditions: $a_{1}=6 \mathrm{AU}, a_{2}=100 \mathrm{AU}$, $i_{1}=65^{\circ}, g_{1}=0^{\circ}, h_{1}=180^{\circ}$. The star has mass $1 M_{\odot}$, the planet has mass $1 M_{J}$, and the outer brown dwarf has mass $40 M_{J}$; colors: time in multiples of $10^{7}$ years; gray indicates that no flip occurred during integration

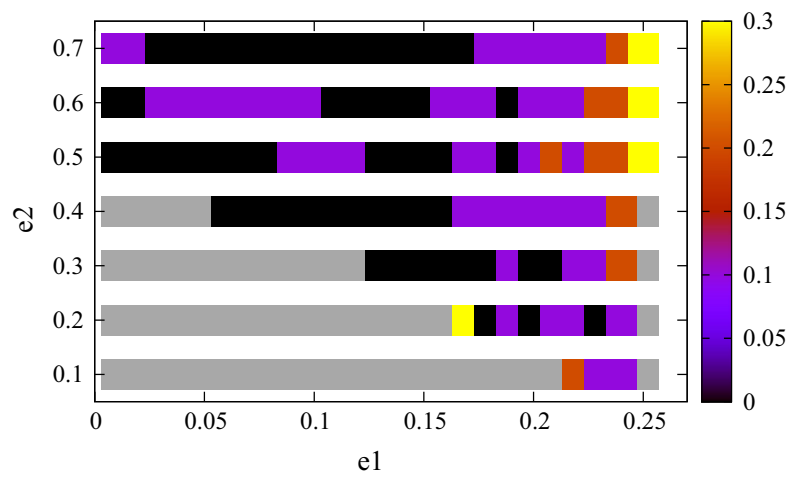

we note that, in general, when the potential up to the fifth order is taken into account, the inclination takes a significantly different time to invert. This is especially true for large values of the eccentricity of the outer orbit $\left(e_{2}=0.5,0.6,0.7\right)$. It is also clear that, with respect to the existence of the flip, there are no differences in including $R_{4 C}$ or $R_{5 C}$ compared to the model that has only $R_{3 C}$. The points in the $e_{1}-e_{2}$ map where there are inversions are the same. The results with the presence of both terms $R_{4 C}$ and $R_{5 C}$ show a reduction in the inversion times compared to the case where only $R_{4 C}$ is considered. Note the presence of a larger black region in Fig. 18 compared to Fig. 19. In general, the maximum difference in the inversion times is approximately $0.3 \times 10^{7}$ years, which represents approximately $3 \%$ of the total integration time. Those analyses are made with respect to the time of the first inversion only, not taking into account oscillations of the inclination.

Figure 20 shows a direct comparison of the model expanded up to $R_{3}$, while Fig. 21 shows the same results for an expansion up to $R_{4}$ and Fig. 22 for an expansion up to $R_{5}$. The differences are again on the order of $3 \%$ in the inversion times in all cases, and no differences regarding the existence or nonexistence of inversions are observed. It is observed that the inclusion of the $R_{4}$ term reduces the difference between analytical and numerical methods for larger values of the eccentricity $e_{2}$. Note the presence of larger black regions for $e_{2} \geq 0.5$. In the opposite sense, the results from the analytical model worsens the quality with the inclusion of the term $R_{4}$ for lower values of $e_{2}$. Note the presence of larger regions of yellow in Fig. 21 compared to Fig. 20.

Extending the comparison to Fig. 22, the same observation is made for larger values of $e_{2}$. The inclusion of the term $R_{5}$ causes an increase in the black regions compared with Figs. 20 
Fig. $20 e_{2} \times e_{1} \times t_{\text {inv }}$.

Difference between direct numerical integration and perturbation potential expanded to third order. Initial conditions: $a_{1}=6 \mathrm{AU}, a_{2}=100 \mathrm{AU}$, $i_{1}=65^{\circ}, g_{1}=0^{\circ}, h_{1}=180^{\circ}$. The star has mass $1 M_{\odot}$, the planet has mass $1 M_{J}$, and the outer brown dwarf has mass $40 M_{J}$; colors: time in multiples of $10^{7}$ years; gray indicates that no flip occurred during integration

Fig. $21 e_{2} \times e_{1} \times t_{\text {inv }}$. Difference between direct numerical integration and perturbation potential expanded to fourth order. Initial conditions: $a_{1}=6 \mathrm{AU}, a_{2}=100 \mathrm{AU}$, $i_{1}=65^{\circ}, g_{1}=0^{\circ}, h_{1}=180^{\circ}$. The star has mass $1 M_{\odot}$, the planet has mass $1 M_{J}$, and the outer brown dwarf has mass $40 M_{J}$; colors: time in multiples of $10^{7}$ years; gray indicates that no flip occurred during integration

Fig. $22 e_{2} \times e_{1} \times t_{\text {inv }}$. Difference between direct numerical integration and perturbation potential expanded to fifth order. Initial conditions: $a_{1}=6 \mathrm{AU}, a_{2}=100 \mathrm{AU}$, $i_{1}=65^{\circ}, g_{1}=0^{\circ}, h_{1}=180^{\circ}$. The star has mass $1 M_{\odot}$, the planet has mass $1 M_{J}$, and the outer brown dwarf has mass $40 M_{J}$; colors: time in multiples of $10^{7}$ years; gray indicates that no flip occurred during integration
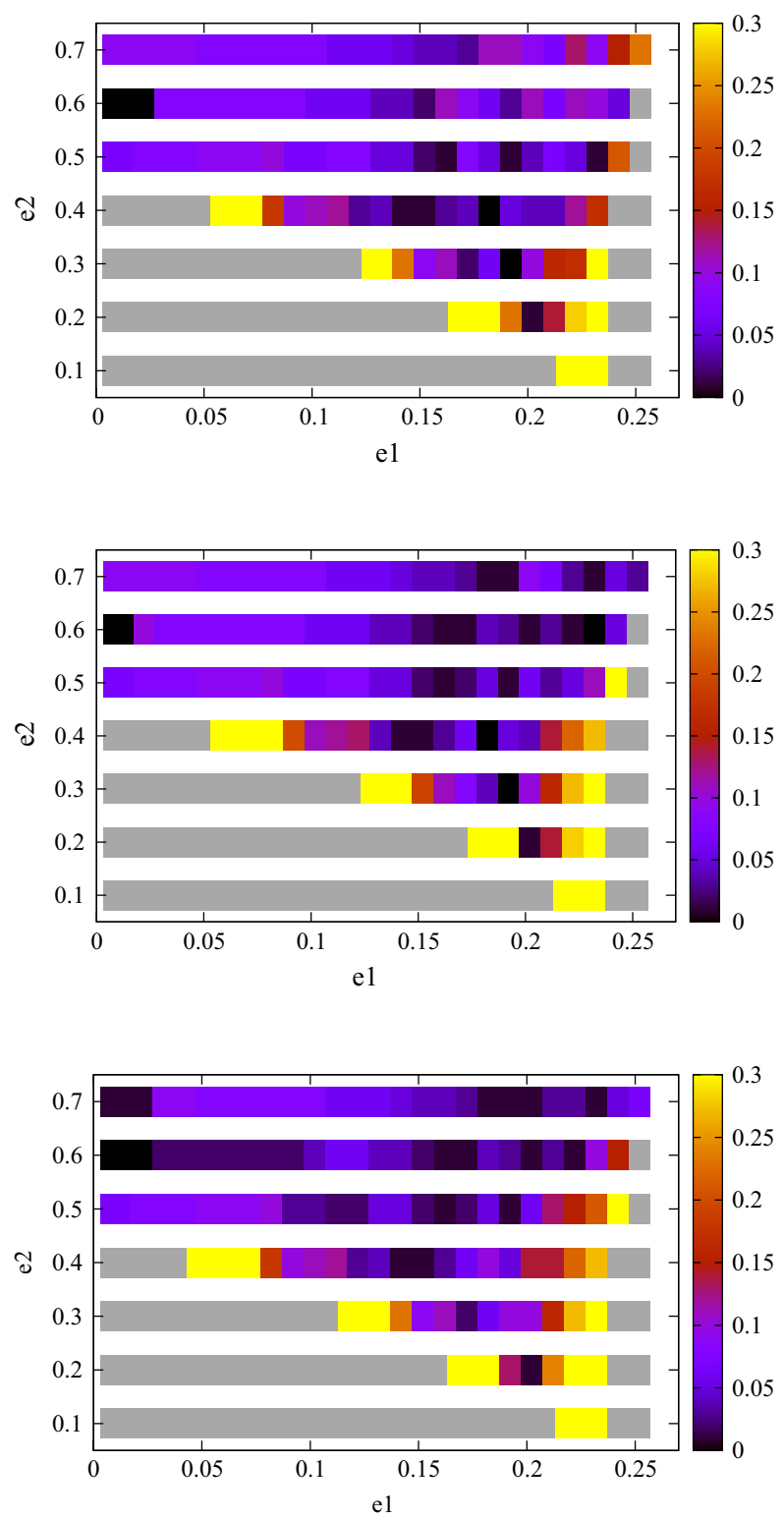

and 21. This means that for large values of $e_{2}$, the quality of the analytical model increases with the order of the expansion, as expected. For lower values of $e_{2}$ the results are about the same as those given by the expansion up to the fourth order. The effects of $e_{1}$ are much smaller because its range is much narrower than that of $e_{2}$.

Thus, the general conclusion is that the analytical model increases its accuracy with the inclusion of higher-order terms, from the point of view of the times for the first flips, at least for values of $e_{2} \geq 0.5$. Those cases have larger perturbations and so require longer expansions. 


\section{Conclusions}

We investigated the secular dynamics of a planet that moves around a central star perturbed by a brown dwarf. We developed the perturbation potential in closed forms up to the third order in a small parameter when the perturbing star is in an elliptical and inclined orbit and up to the fifth order in a small parameter when the outer orbit is elliptical, planar, and fixed in space. We analyzed the effects of the potential in different orders. The dynamics is strongly modified compared with the Kozai-Lidov classic problem, where only up to the second order is considered in the expansion of the potential. We compared the secular evolution of systems with and without the third-, fourth-, and fifth-order terms of the perturbation potential. Here the $\sum_{j=2}^{5} R_{j C}$ terms of the perturbation potential are developed without eliminating the nodes before deriving the equations of motion. We also performed full numerical integrations using the Bulirsch-Stoer method available in the Mercury package for comparison with the analytical model. The findings of this paper are as follows:

1. The perturbation potential is developed in closed form up to the third order in a small parameter when the perturbing star is in an elliptical and inclined orbit. In this case, the inner and outer orbits are mutually perturbed. Here, the elimination of nodes was not carried out before the derivation of the equations of motion, as is commonly done in the literature, in cases where the Hamiltonian formalism is used. Thus, the perturbation potential presented in this research contains terms that would be discarded if the nodes were eliminated at the level of the Hamiltonian. When the inner and outer orbits are mutually perturbed, even observing that the perturbing star is much less perturbed than the planet's orbit, the result is in agreement with the direct numerical integration of the problem of three bodies.

We show the dependence of the first flip on the semimajor axis and eccentricity. In general, larger values of $e_{2}$ reduce the time required for the first inversion for low values of the semimajor axis of the perturbing star. There is a limit below which the inversion occurs and above which inversions no longer occur (Fig. 6) for times up to $10^{7}$ years. As an example, for a semimajor axis of $100 \mathrm{AU}$, inversion occurs only for eccentricities equal to 0.5 and 0.6 or larger. Therefore, when considering distant stars, such as a hierarchical triple system, inversion occurs only for large values of eccentricity of the perturbing star's orbit. We also show that the time for the first inversion increases when the semimajor axis increases. The occurrence of this phenomenon depends on the semimajor axis and eccentricities of the perturbing star. This semimajor axis also has a strong effect on the phenomenon of inversion since the closer the perturbed planet is to the perturbing star, the larger the effect is. We show orbits that librate around the equilibrium point for $g_{1}=90^{\circ}$ or $g_{1}=270^{\circ}$. The orbits are described by regions rather than lines, as is common in the literature. This happens because the inclination flips between prograde and retrograde orbits.

2. We developed the perturbation potential in closed form up to the fifth order in a small parameter when the perturbing star orbit is elliptical, planar, and fixed in space. When the equations of motion are developed explicitly up to the fifth order, we show the importance of the $R_{4}$ and $R_{5}$ terms in changing the inversion times of the flip, i.e., the time where the inclination of the inner planet can flip from prograde to retrograde trajectories.

When considering that only the internal orbit is perturbed and the perturbation potential expands up to the third order, the planet inclination changes the inversion time compared to the numerical results, after the third inversion. When considering up to the fourth order, the inclination behavior differs from the numerical result as early as the second inversion. Now, assuming that the potential expands up to the fifth order, the analytical model is in agreement with the results of the numerical simulations and agrees with the case when one 
considers in the disturbing potential up to the third-order, for the case where the two orbits are mutually perturbed and inclined. When considering the perturbation potential up to the fifth order, the planet inclination behavior differs slightly from the results of the direct numerical integrations starting at the third inversion, but this difference is very small compared to those that occur with the perturbation potential developed up to the third or fourth order. Thus, one realizes that the term $R_{5 C}$ contributes to the results, and the equations developed here can reproduce more faithfully the results found in the direct numerical integration of the three-body problem. The results show that the inclusion of the $R_{4}$ term gives results that are worse than those where the expansion is stopped in the $R_{3}$ term, but the inclusion of the $R_{5}$ term corrects and improves the results. Note also that the odd terms of the expansion in the Legendre polynomial are strongly dominant with respect to the terms of even order.

Acknowledgments Sponsored by $\mathrm{CNPq}-\mathrm{Brazil}$. The authors are grateful to $\mathrm{CNPq}$ (National Council for Scientific and Technological Development)-Brazil for contracts 306953/2014-5, 304700/2009-6, and 303070/2011-0, FAPESP (Foundation to Support Research in São Paulo State) SP-Brazil under Contracts 2011/08171-3, 2011/05671-5, 2012/21023-6, and 2014/06688-7 and CAPES. We thank Dr. Denilson Paulo Souza dos Santos and Dr. André Izidoro Ferreira da Costa for help with some Maple commands.

\section{References}

Albrecht, S., Winn, J.N., Johnson, J.A., et al.: Obliquities of Hot Jupiter host stars: evidence for tidal interactions and primordial misalignments. Astrophys. J. 757(1), 18 (2012)

Antonini, F., Murray, N., Mikkola, S.: Black hole triple dynamics: breakdown of the orbit average approximation and implications for gravitational wave detections. Astrophys. J. 781(1), 45 (2014)

Beaugé, C., Ferraz-Mello, S., Michtchenko, T.A.: Multi-planet extrasolar systems detection and dynamics. Res. Astron. Astrophys. 12(8), 1044-1080 (2012)

Bode, J.N., Wegg, C.: Production of EMRIs in supermassive black hole binaries (2013) arXiv:1310.5745v1

Brouwer, D.: Solution of the problem of an artificial satellite, theory without drag. Astron. J. 64(9), 378-397 (1959)

Brouwer, D., Clemence, G.M.: Methods of Celestial Mechanics. Academic Press, New York (1961)

Carvalho, J.P.S., Vilhena de Moraes, R., Prado, A.F.B.A., Winter, O.C.: Analysis of the secular problem for triple star systems. J. Phys. Conf. Ser. 465, 1-6 (2013). doi:10.1088/1742-6596/465/1/012010

Chambers, J.E.: A hybrid symplectic integrator that permits close encounters between massive bodies. Mon. Not. R. Astron. Soc. 304, 793-799 (1999)

Correia, A.C.M., Laskar, J., Farago, F., Boué, G.: Tidal evolution of hierarchial and inclined systems. Celest. Mech. Dyn. Astron. 111, 105-130 (2011)

Correia, A.C.M., Boué, G., Laskar, J.: Pumping the eccentricity of exoplanets by tidal effect. Astrophys. J. Lett. 744(L23), 1-5 (2012)

Ford, E.B., Kozinsky, B., Rasio, F.A.: Secular evolution of hierarchical triple star systems. Astrophys. J. 535, 385-401 (2000)

Ford, E.B., Kozinsky, B., Rasio, F.A.: ERRATUM: "Secular evolution of hierarchical triple star systems" (Astrophys. J. 535, 385 [2000]). Astrophys. J. 605, 966 (2004)

Harrington, R.: The stellar three-body problem. Celest. Mech. 1, 200-209 (1969)

Hori, G.: The motion of a hyperbolic artificial satellite around the Oblate Earth. Astron. J. 66(6), 258-263 (1961)

Jefferys, W.H., Moser, J.: Quasi-periodic solutions for the three-body problem. Astron. J. 71(7), 568-578 (1966)

Katz, B., Dong, S., Malhotra, R.: Long-term cycling of Kozai-Lidov cycles: extreme eccentricities and inclinations excited by a distant eccentric perturber. Phys. Rev. Lett. 107, 181101 (2011)

Kovalevsky, J.: Introduction to Celestial Mechanics. D. Reidel, Dordrecht (1967)

Kozai, Y.: Secular perturbations of asteroids with high inclination and eccentricity. Astron. J. 67(9), 591 (1962)

Krymolowski, Y., Mazeh, T.: Studies of multiple stellar-II. Second-order averaged hamiltonian to follow long-term orbital modulations of hierarchical triple systems. Mon. Not. R. Astron. Soc. 304, 720-732 (1999) 
Laskar, J., Boué, G.: Explicit expansion of the three-body disturbing function for arbitrary eccentricities and inclinations. Astron. Astrophys. 522(A60), 1-11 (2010)

Lee, M.H., Peale, S.J.: Secular evolution of hierarchical planetary systems. Astrophys. J. 592, 1201-1216 (2003)

Li, G., Naoz, S., Holman, M., Loeb, A.: Chaos in the test particle eccentric Kozai-Lidov mechanism. Astrophys. J. 791(86), 1-10 (2014a)

Li, G., Smadar Naoz, S., Kocsis, B., Loeb, A.: Eccentricity growth and orbit flip in near-coplanar hierarchical three-body systems. Astrophys. J. 785:116, 1-8 (2014b)

Lidov, M.L.: The evolution of orbits of artificial satellites of planets under the action of gravitational perturbations of external bodies. Planet. Space Sci. 9, 719-759 (1962)

Lithwick, Y., Naoz, S.: The eccentric Kozai mechanism for a test particle. Astrophys. J. 742(94), 1-8 (2011)

Naoz, S., Farr, W.M., Lithwick, Y., Rasio, F.A., Teyssandier, J.: Hot jupiters from secular planet-planet interations. Nature 473, 187-189 (2011)

Naoz, S., Farr, W.M., Lithwick, Y., Rasio, F.A., Teyssandier, J.: Secular dynamics in hierarchical three-body systems. MNRAS 431, 2155-2171 (2013)

Naoz, S., Farr, W.M., Rasio, F.A.: On the formation of hot jupiters in stellar binaries. Astrophys. J. Lett. 754(L36), 1-6 (2012)

Petrovich, C.: Hot jupiters from coplanar high-eccentricity migration. Astrophys. J. 805(1), 75 (2015a)

Petrovich, C.: Steady-state planet migration by the Kozai-Lidov mechanism in stellar binaries. Astrophys. J. 799(1), 27 (2015b)

Prado, A.F.B.A.: Third-body perturbation in orbits around natural satellites. J. Guid. Control Dyn. 26(1), 33-40 (2003)

Takeda, G., Kita, R., Rasio, F.A.: Planetary systems in binaries. I. Dynamical classification. Astron. J. 683, 1063-1075 (2008)

Tremaine, S., Touma, J., Namouni, F.: Satellite dynamics on the laplace surface. Astron. J. 137, 37063717 (2009)

Triaud, A.H.M.J., Collier, C.A., Queloz, D., et al.: Spin-orbit angle measurements for six southern transiting planets. New insights into the dynamical origins of hot Jupiters. Astron. Astrophys. 524(A25), 1-10 (2010)

Valtonen, M., Karttunen, H.: The three-body problem. Cambridge University Press, Cambridge (2006)

Yokoyama, T., Vieira Neto, E., Winter, O. C., Sanchez, D. M., Brasil, P.I.O.: On the evection resonance and its connection to the stability of outer satellites. Math. Probl. Eng. Article ID 251978, 16 (2008). doi:10. $1155 / 2008 / 251978$

Yokoyama, T., Santos, M.T., Gardin, G., Winter, O.C.: On the orbits of the outer satellites of Jupiter. Astron. Astrophys. 401, 763-772 (2003) 